Research Paper

\title{
Triple-Modal Imaging of Magnetically-Targeted Nanocapsules in Solid Tumours In Vivo
}

\author{
Jie Bai ${ }^{1}$, Julie T.-W. Wang ${ }^{1}$, Noelia Rubio ${ }^{1}$, Andrea Protti², Hamed Heidari ${ }^{3}$, Riham Elgogary ${ }^{4}$, Paul \\ Southern', Wafa' T. Al-Jamal', Jane Sosabowski ${ }^{7}$, Ajay M. Shah², Sara Bals ${ }^{3}$, Quentin A. Pankhurst ${ }^{5}$, \\ Khuloud T. Al-Jamal ${ }^{\square}$ \\ 1. Institute of Pharmaceutical Science, Faculty of Life Sciences \& Medicine, King's College London, London, SE1 9NH, UK \\ 2. Cardiovascular Division, James Black Centre, King's College London British Heart Foundation Centre of Excellence, London, SE5 9NU, UK \\ 3. Electron Microscopy for Materials Research (EMAT), University of Antwerp, Groenenborgerlaan 171, B-2020, Antwerp, Belgium \\ 4. Faculty of Pharmacy, Ain Shams University, Khalifa El-Maamon Street, Abbasiya Square, Cairo, 11566, Egypt \\ 5. UCL Healthcare Biomagnetics Laboratory, University College London, 21 Albemarle Street, London, W1S 4BS, UK \\ 6. School of Pharmacy, University of East Anglia, Norwich Research Park, Norwich, NR4 7TJ, UK \\ 7. Centre for Molecular Oncology, Bart's Cancer Institute, Queen Mary University of London, London, EC1M 6BQ, UK
}

$\triangle$ Corresponding author: Institute of Pharmaceutical Science, Faculty of Life Sciences \& Medicine, King's College, London, London, SE1 9NH, UK. Phone: +44(0)20 7848 4525; E-mail: khuloud.al-jamal@kcl.ac.uk

(C) Ivyspring International Publisher. Reproduction is permitted for personal, noncommercial use, provided that the article is in whole, unmodified, and properly cited. See http://ivyspring.com/terms for terms and conditions.

Received: 2015.02.17; Accepted: 2015.04.22; Published: 2016.01.01

\begin{abstract}
Triple-modal imaging magnetic nanocapsules, encapsulating hydrophobic superparamagnetic iron oxide nanoparticles, are formulated and used to magnetically target solid tumours after intravenous administration in tumour-bearing mice. The engineered magnetic polymeric nanocapsules $m$-NCs are $\sim 200 \mathrm{~nm}$ in size with negative Zeta potential and shown to be spherical in shape. The loading efficiency of superparamagnetic iron oxide nanoparticles in the $\mathrm{m}-\mathrm{NC}$ was $\sim 100 \%$. Up to $\sim 3$ - and $\sim 2.2$-fold increase in tumour uptake at 1 and $24 \mathrm{~h}$ was achieved, when a static magnetic field was applied to the tumour for 1 hour. $m$-NCs, with multiple imaging probes (e.g. indocyanine green, superparamagnetic iron oxide nanoparticles and indium-111), were capable of triple-modal imaging (fluorescence/magnetic resonance/nuclear imaging) in vivo. Using triple-modal imaging is to overcome the intrinsic limitations of single modality imaging and provides complementary information on the spatial distribution of the nanocarrier within the tumour. The significant findings of this study could open up new research perspectives in using novel magnetically-responsive nanomaterials in magnetic-drug targeting combined with multi-modal imaging.
\end{abstract}

Key words: PEGylated PLGA, magnetic targeting, optical imaging, nuclear imaging, magnetic resonance imaging

\section{Introduction}

Superparamagnetic iron oxide nanoparticles (SPIONs) have been investigated for biomedical applications. $[1,2]$ This superparamagnetic property is ideal for in vivo use since SPIONs can be highly magnetised and literally drag drug molecules to their target site in the body under the influence of an applied magnet field whereas zero remanent magnetisation retained after the removal of magnetic field.[2, 3] SPIONs are frequently used for magnetic drug targeting which is regarded as a physical targeting method and does not require chemical conjugation of targeting ligands.[4] Targeting therapeutic drugs with magnetic forces has been proposed to concentrate the therapeutic agent at the target site thus lowering the administered dosage and reducing systemic side effects.[5]

A number of imaging techniques are being applied for biomedical applications. This includes magnetic resonance (MR) imaging, nuclear imaging, optical imaging, ultrasound imaging, etc. The emerging field of multi-modal imaging using multi-labelled imaging probes promises to image the 
same organ with multiple imaging techniques since each imaging technique has its own unique advantages and intrinsic limitation.[6-15] For example, MR imaging can offer high spatial resolution (down to 50-250 $\mu \mathrm{m}$ ) and the capacity to simultaneously obtain physiological and anatomical information, but sensitivity of MRI is a limiting factor.[16] Optical imaging allows for rapid screening and thus is highly desirable in clinical and pre-clinical use[17], but is limited by its penetration depth in tissues and therefore is considered to be semi-quantitative. Nuclear imaging offers excellent sensitivity (down to $10^{-10}$ to $10^{-12} \mathrm{~mol} / \mathrm{L}$ ) and exhibits no limitation for tissue penetration.[16, 18] However, image resolution is restricted to the millimetre level.[19, 20] Therefore, multi-modal imaging systems are required since performing cross-modality validation can provide more accurate and reliable data than when single imaging modality is used.[21-23]

In this work, we developed a novel approach using polymeric nanocapsule to engineer a triple-modal fluorescence/MR/SPECT imaging system and investigated their passive and magnetic targeting properties for cancer imaging. To our knowledge, this is the first report to examine the magnetic tumour targeting of magnetic nanocarrier using triple-imaging technique. In this study, SPION and a near-infrared dye, indocyanine green (ICG), were physically incorporated in the magnetic nanocapsules as a negative MR contrast agent and fluorescence imaging probe, respectively. Furthermore, a chelating agent, diethylene triamine pentaacetic acid (DTPA), was chemically conjugated to the PEGylated PLGA polymer for radio-isotope chelation and therefore facilitated nuclear imaging. In vivo studies were performed to characterise the organ biodistribution profile and magnetic targeting of the magnetic nanocapsules using triple-modal imaging technique.

\section{Results and discussion}

\section{Formulation and characterisation of magnetic nanocapsules}

Nanocapsules formulations prepared include PEGylated poly lactic-co-glycolic acid (PLGA) non-magnetic nanocapsules (NC) and magnetic nanocapsules $(m-N C)$. PEGylated PLGA ${ }_{18 K D a}$ conjugate was synthesised and characterised by FT-IR and ${ }^{1}$ H-NMR (Additional File 1: Fig. S1). Both formulations were prepared by single emulsification/solvent evaporation method (Scheme 1). The hydrodynamic size obtained by dynamic light scattering was in range of $203 \pm 4 \mathrm{~nm}$ (Table 1). The inclusion of SPION in $m$-NC did not lead to statistically significant difference in NC diameter ( $205 \pm 3 \mathrm{~nm}, \mathrm{p}>0.05)$, however, it resulted in a decrease in Zeta potential of NC from -45 $\pm 2 \mathrm{mV}$ to $-36 \pm 1 \mathrm{mV}(\mathrm{p}<0.01)$ (Table 1$)$. The reduction in the overall negative charge of $m$-NC might be attributed to hydrophobic interactions between the oleic acid coatings of SPIONs and the negatively charged PLGA polymeric shell. PLGA is FDA approved and exhibits high lipophilicity so is ideal to accommodate hydrophobic SPIONs coated with oleic acid. SPION encapsulation efficiency (EE \%) was quantified by inductively couple plasma mass spectrometry (ICP-MS) after purification by size exclusion chromatography. ICP-MS results suggested the SPION loading to be $95 \% \pm 3 \%$ (equivalent to $1.76 \%, \mathrm{w} / \mathrm{w}$ SPION $/ m-N C$, Table 1). This was further confirmed by thermogravimetric analysis (TGA) with \% weight remaining of $1.84 \%$ at $600{ }^{\circ} \mathrm{C}$ (Additional File 1: Fig. S2a).

Table 1. Physicochemical characterisation of PEGylated NCs prepared by single emulsification/solvent evaporation method

\begin{tabular}{|c|c|c|c|c|c|}
\hline Formulation & $\begin{array}{l}\text { Initial SPION } \\
\text { loading ( } \% \\
\text { w/w, } \\
\text { SPION/NC) }\end{array}$ & $\begin{array}{l}\text { Hydrodynamic } \\
\text { size } \pm \text { SD }(\mathrm{nm}) \\
\dagger\end{array}$ & $\mathrm{PDI} \pm \mathrm{SD} \dagger$ & $\begin{array}{l}\text { Zeta } \\
\text { potential } \\
\pm S D \\
(\mathrm{mV}) \ddagger\end{array}$ & $\begin{array}{l}\text { SPION } \\
\text { EE } \% \pm \\
\text { SD\$ }\end{array}$ \\
\hline $\mathrm{NC}$ & - & $203 \pm 4$ & $0.12 \pm 0.01$ & $-45 \pm 2$ & - \\
\hline$m$-NC & $1.84 \%$ & $205 \pm 3$ & $0.16 \pm 0.01$ & $-36 \pm 1$ & $95 \pm 3$ \\
\hline
\end{tabular}

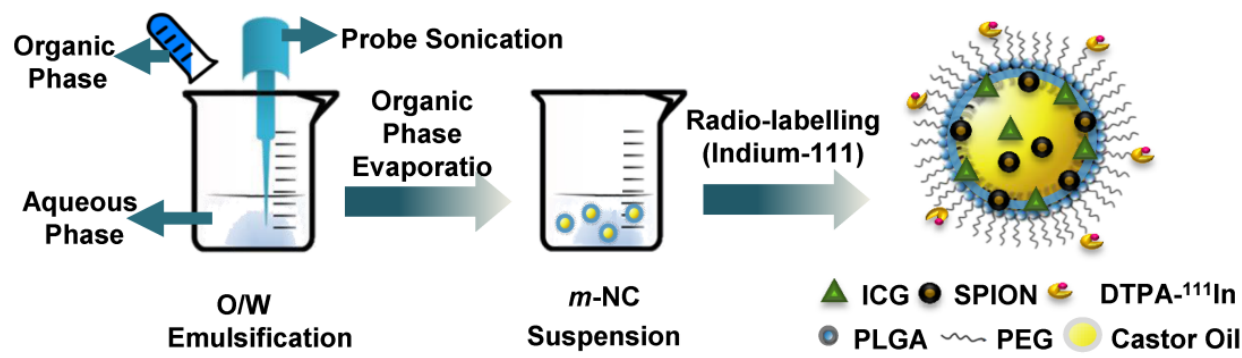

Scheme 1. Preparation of $\boldsymbol{m}$-NC with triple-modal imaging probes by single emulsification/solvent evaporation method. The $m$-NC was formulated by the emulsification of an organic phase (dichloromethane) containing polymer, oil, lipophilic surfactant, SPION and ICG and an aqueous phase with hydrophilic surfactant using probe sonication. Radio-labelling of indium-111 with $m-\mathrm{NC}$ was carried out after its formation. 
The morphology of NCs was examined initially by atomic force microscopy (AFM). As shown in Fig. 1a, NCs and $m$-NCs were spherical in shape and showed a homogeneous size distribution. The phase image allows distinguishing different surface features and provides significantly more contrast than the topographic images, therefore can be useful for compositional mapping of surfaces and interfaces of polymeric materials.[24] In Fig. 1a, a halo in the periphery of both types of NCs observed in phase images suggests that the presence of different materials therefore indicating the formation of core-shell structure. We reported similar observations previously. [25] No difference was observed in NC morphology with the inclusion of SPION in terms of size and surface features. Low-dose cryo transmission electron microscopy (cryo-TEM) was employed to investigate the internal structure of $m$-NCs. Both 2D projections and $3 \mathrm{D}$ reconstruction suggested that the SPION (electron-dense nanoparticle) was homogenously encapsulated in the $m$-NC (Fig. 1b, Additional File 2: Movie S1).

The magnetic properties of SPION and $m$-NC were assessed by measuring the magnetic hysteresis curves (Additional File 1: Fig. S2b). The saturation magnetisation (Ms) value was ca. $72 \mathrm{emu} / \mathrm{g}$ Fe for both SPION and $m$-NC when normalised to the same iron content. The coercive field (Hc) was less than 1 Oe, which is within experimental error of zero, hence confirming the superparamagnetic (anhysteretic) character of both samples. a
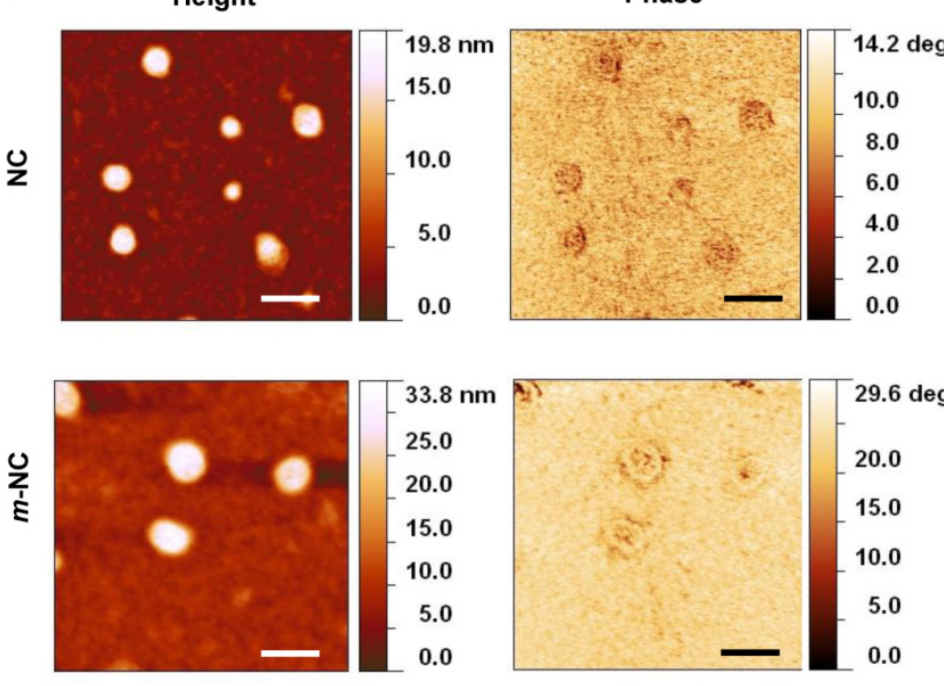

Phase

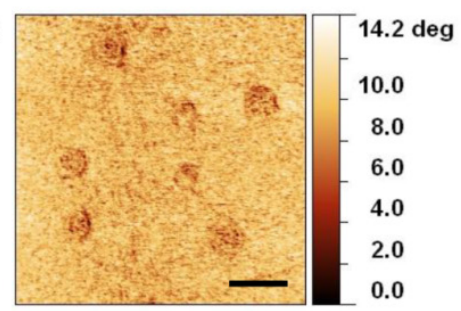

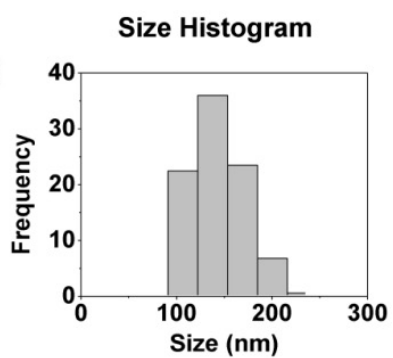

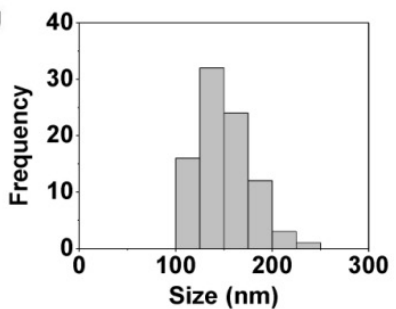

b
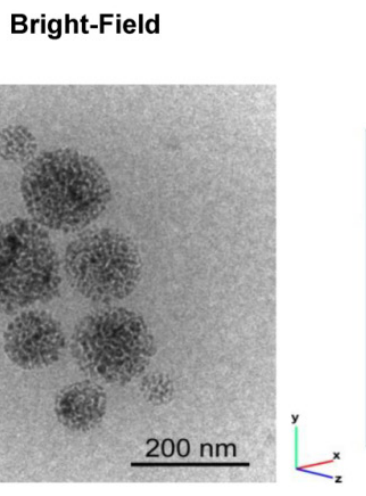

3D Tomographic

Reconstruction

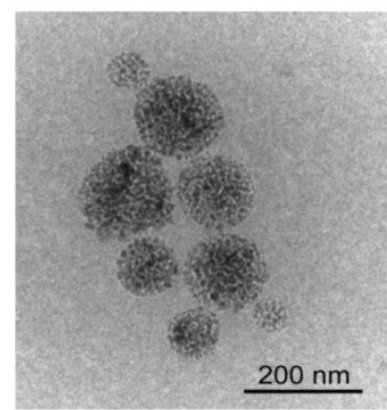

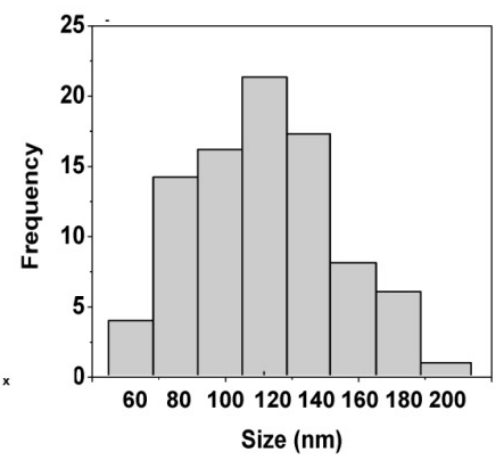

Figure 1. Morphological examination of $m-N C$ and NC. a, AFM images of NC and $m$-NC prepared by single emulsification/solvent evaporation method. The height, phase images and size histograms of NC and $m-\mathrm{NC}$ are presented. Samples were prepared and diluted to $50 \mu \mathrm{g}$ polymer/ml and freeze-dried on mica slices before imaging. All images were acquired while operating in a tapping mode under ambient conditions. A spherical shape was observed in both types of NC with average diameters of $152 \pm 25 \mathrm{~nm}(\mathrm{n}=95)$ and $149 \pm 30 \mathrm{~nm}(\mathrm{n}=91)$ obtained for NC and $m-\mathrm{NC}$, respectively. Scale bars are $200 \mathrm{~nm}$. b, Cryo-TEM images of $m-\mathrm{NC}$ prepared by single emulsification/solvent evaporation method. The CryoTEM projection image, yx and yz orthogonal slices through the 3D tomographic reconstruction of the vitrified sample and a histogram of the size distribution of $m-\mathrm{NC}$ is presented. CryoTEM measurements demonstrated an average size of $122 \pm 4 \mathrm{~nm}$ for $m$-NC. 


\section{In vivo optical imaging with magnetic targeting}

A near infrared dye, indocyanine green (ICG), was incorporated to the NC and $m-\mathrm{NC}$ at $0.075 \% \mathrm{w} / \mathrm{w}$ ICG/NC. No significant change in hydrodynamic size and Zeta potential of NC was observed with the encapsulation of ICG (Table 2). To characterise the optical properties of $m$-NC/NC-ICG, the fluorescence of ICG was tested on a well plate at different concentrations in presence of serum. The incubation of $m-\mathrm{NC}$ with serum showed no influence on ICG fluorescence intensity and no quenching effect was seen up to 30 $\mu \mathrm{g} / \mathrm{ml}$ ICG concentration (Additional File 1: Fig. S3a-c). In addition, no significant drop in fluorescence intensity was observed when $1.85 \%$ SPION (w/w, $\mathrm{SPION} / m-\mathrm{NC}$ ) was incorporated compared to NC-ICG, however, increasing SPION loading to $7.02 \%$ $\mathrm{w} / \mathrm{w}$ caused approximately $41 \%$ reduction in fluorescence (Additional File 1: Fig. S3d). Quenching of fluorescent imaging probes due to the presence of SPION has been reported previously.[26, 27] As a result, $1.85 \%$ SPION (w/w, SPION $/ m-\mathrm{NC})$ was used for the in vivo triple-modal imaging studies.
Table 2. Physicochemical characterisation of ICG encapsulated NCs prepared by single emulsification/solvent evaporation method

\begin{tabular}{lllll}
\hline $\begin{array}{l}\text { Formulation } \\
\text { Hydrodynamic } \\
\text { size } \pm \text { SD }(\mathbf{n m}) \dagger\end{array}$ & PDI \pm SD $\dagger$ & \multicolumn{2}{l}{$\begin{array}{l}\text { Zeta potential } \pm \text { ICG EE } \% \pm \\
\text { SD }(\mathbf{m V}) \ddagger\end{array}$} & SD $^{\$}$ \\
\hline NC-ICG & $210 \pm 8$ & $0.12 \pm 0.01$ & $-42 \pm 1$ & $98.95 \pm 0.63$ \\
$m$-NC-ICG & $207 \pm 4$ & $0.16 \pm 0.01$ & $-34 \pm 1$ & $98.75 \pm 0.35$ \\
\hline †Size was measured by dynamic light scattering in deionised water $(\mathrm{n}=3)$ \\
₹ Values were obtained in deionised water $(\mathrm{n}=3)$ \\
\$EE\% was determined by fluorescence spectroscopy $(\mathrm{n}=3)$
\end{tabular}

$m$-NC-ICG was intravenously injected into a CT26 tumour-bearing balb/c mice and free ICG in PBS was injected as a control. Pronounced signals of $m$-NC-ICG were seen over the whole body in Fig. 2a, indicating prolonged blood circulation. Tumour accumulation was observed at $24 \mathrm{~h}$ post-injection in live animals and ex vivo imaging (Fig. 2b). On the contrary, the mice injected with free ICG showed rapid clearance from the body and no sign of tumour accumulation. The distinct difference in organ biodistribution profiles between the free and encapsulated ICG suggested the stable encapsulation of ICG in $m$-NC.

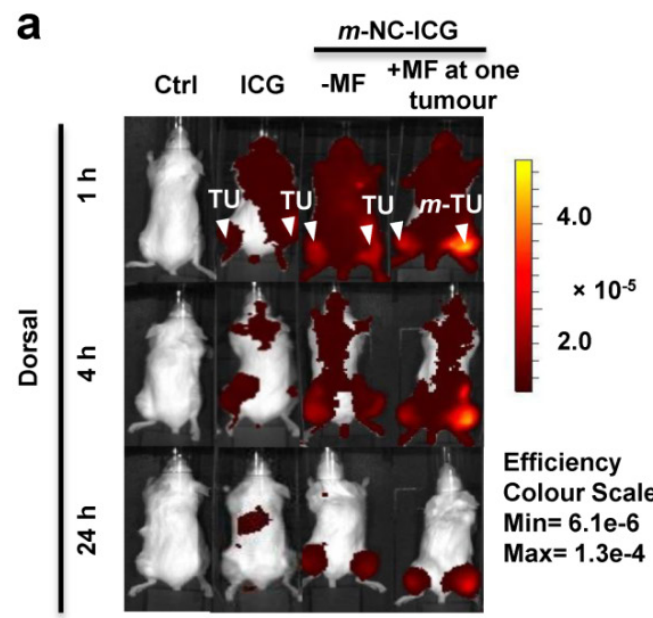

b
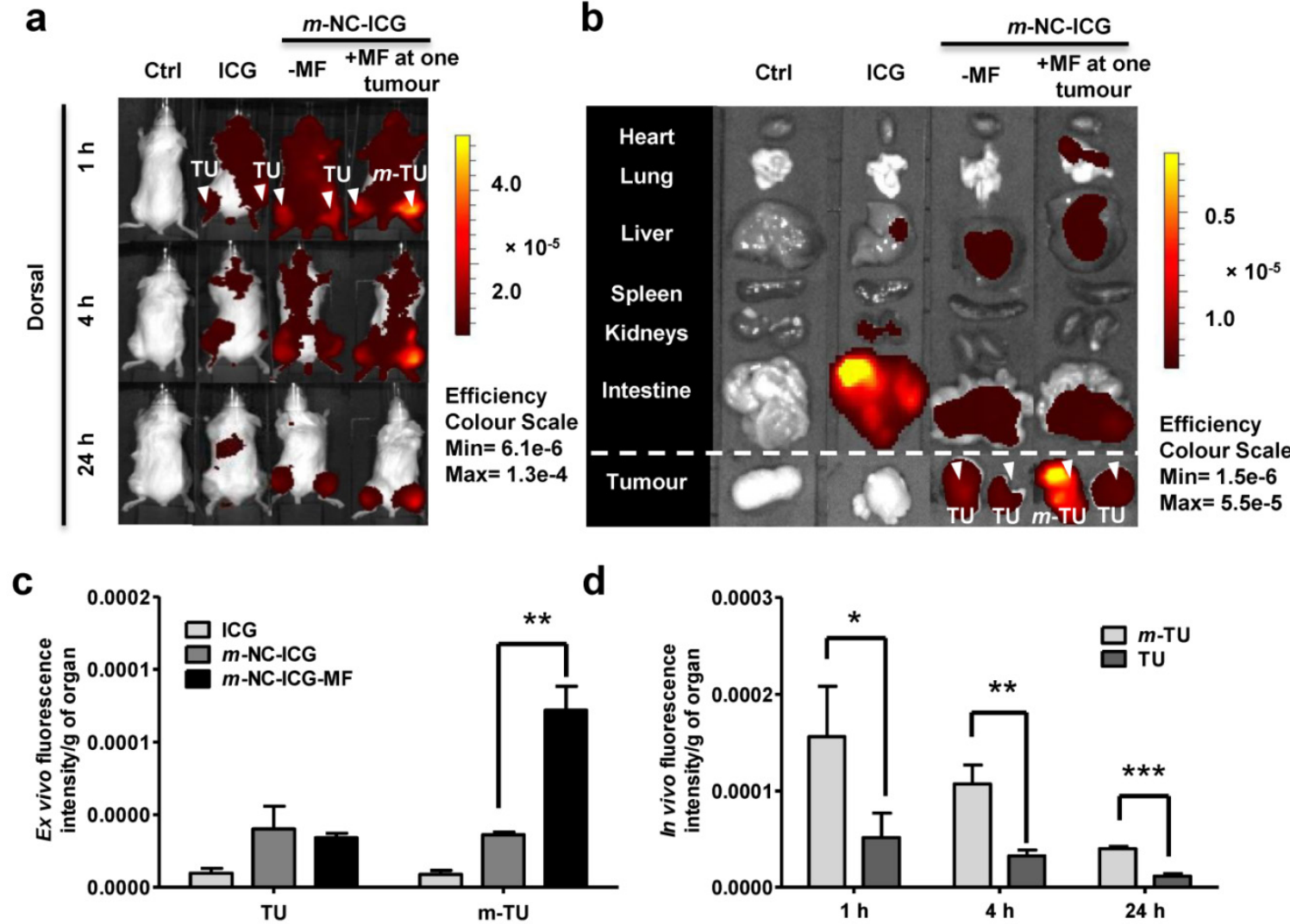

Figure 2. In vivo and ex vivo imaging of ICG-labelled $m$-NC in CT26 tumour-bearing balb/c mice under the influence of magnetic field. Mice were i.v. injected with PBS, ICG or $m$-NC-ICG, respectively. Magnetic field (MF) was applied by placing a permanent magnet $(0.515 \mathrm{~T})$ at one tumour $(\mathrm{m}$-TU) for $1 \mathrm{~h}$. a, Representative whole body in vivo images of mice obtained at 1, 4 and $24 \mathrm{~h}$ post-injection. b, Representative ex vivo images of excised organs at $24 \mathrm{~h}$ post-injection. c, Ex vivo quantification of fluorescence signals of ICG and ICG-labelled $m$-NC per gram of tumour at $24 \mathrm{~h}$. d, In vivo quantification of fluorescence signals of ICG per gram of tumour at the magnetically targeted site $(m-T U)$ and the non-magnetically targeted site (TU) of mice at different time points. Tumour accumulation in b was observed with mice injected with $m-N C$-ICG, but not with free ICG which exhibited high signals in intestine. Quantitative results also showed significantly enhanced uptake in $m-T U$ compared to TU $(* p<0.05$, $* * p<0.01$, *** $p<0.005)$. Values were expressed as means \pm SD $(n=3)$. All images were obtained by IVIS Lumina ${ }^{\circledR}$ III and data were analysed by Living Image ${ }^{\circledR} 4.3 .1$ Service Pack 2 software. 
Encouraged by the biodistribution and tumour accumulation studies of $m$-NC-ICG, we then investigated the magnetic targeting of $m$-NC in the same tumour model and tumour uptake was monitored in vivo by optical imaging. A permanent magnet $(0.515$ $\mathrm{T})$ was placed at one tumour site $(m-\mathrm{TU})$ for $1 \mathrm{~h}$. Contra-lateral tumour (TU) was used as an internal control where no magnet was applied. Interestingly, significantly enhanced fluorescence signals at $m$-TU were seen at $1 \mathrm{~h}$ immediately after the removal of magnet and the increased signals were retained in the tumour up to $24 \mathrm{~h}$ (Fig. 2a). Ex vivo organ images and quantification showed that nearly 3 -fold increase in fluorescence/g tumour was seen in $m$-TU compared to TU while no significant changes were seen in other organs (Fig. 2c and d).

\section{Whole body SPECT/CT imaging and gamma scintigraphy}

Optical imaging is considered to be a semi-quantitative technique due to its limited penetration depth in the body. Thus, to assess the pharmacokinetic profile and organ biodistribution of $m$-NC quantitatively, SPECT/CT imaging followed by ex vivo gamma scintigraphy was performed. The $m$-NC formulation was modified to incorporate PLGA $_{18 \mathrm{KDa}}-\mathrm{PEG}_{3.5 \mathrm{KDa}}-\mathrm{DTPA}$ at $10 \% \mathrm{w} / \mathrm{w}$ of total polymer content. Both NC and $m$-NC showed $100 \%$ radio-labelling efficiency with indium-111 and good radio-labelling stability of indium-111 labelled m-NC in PBS and 50\% serum was also obtained (Fig. 3a and b). NC and $m$-NC without addition of $\mathrm{PLGA}_{18 \mathrm{KDa}}-\mathrm{PEG}_{3.5 \mathrm{KDa}}-\mathrm{DTPA}$ were included as controls and less than 3\% labelling efficiency was seen with both types of NCs, indicating the radio-labelling of indium-111 was due to the chelation with DTPA molecule and it was not affected by the presence of SPION (Fig. 3a).
SPECT/CT imaging was performed after i.v. administration of ${ }^{111}$ In labelled $m$-NC (approximately 8-10 MBq per injection) in CT26 tumour-bearing mice. Three-dimensional reconstruction of the whole animal by SPECT/CT imaging was conducted immediately after injection ( $<30 \mathrm{~min}$ ), at $4 \mathrm{~h}$ and $24 \mathrm{~h}$ post-injection (Additional File 1: Fig. S4a). Despite the intense radio-activity signals in heart and major blood vessels at 0-30 min and $4 \mathrm{~h}$, tumour accumulation started to appear at $4 \mathrm{~h}$ post injection (Fig. S4a, dashed square) and became clearer at $24 \mathrm{~h}$ post injection due to the enhanced permeability and retention (EPR) effect. As expected, predominate tissue accumulation in liver and spleen was also seen due to the RES uptake at $24 \mathrm{~h}$. Intense signals were detected in kidneys and bladder of mice within $30 \mathrm{~min}$ and at $4 \mathrm{~h}$ post-injection, indicating the renal clearance of constituting polymers.

Quantitative studies by gamma scintigraphy were performed in parallel to SPECT/CT imaging at 1,4 and $24 \mathrm{~h}$ post-injection of $m$-NC. In blood clearance profiles (Fig. S4b), $57.0 \pm 13.3 \%$ ID of $m$-NC-111In was detected in blood at $1 \mathrm{~h}$ post-injection and the values dropped to $26.8 \pm 7.9 \%$ and $4.46 \pm 0.6 \%$ ID after 4 and $24 \mathrm{~h}$, respectively. Prolonged blood circulation time is considered to be a pre-requisite for EPR effect and good tumour uptake.[28] Accumulation of $m$-NC in heart, lung and kidneys decreased over $24 \mathrm{~h}$ while increasing accumulations were seen in liver and spleen with $7.37 \pm 0.24 \%$ and $12.97 \% \pm 0.5 \% \mathrm{ID} / \mathrm{g}$ at 24 $h$, respectively. Negligible radio-activity $(\sim 0.15 \%$ ID/g) was measured in brain at $24 \mathrm{~h}$. Tumour uptake of $m$-NC increased from $1.00 \pm 0.02 \%(1 \mathrm{~h})$ to $3.62 \pm$ $1.42 \%$ (24 h) ID/g of tumour (Fig. S4d). The quantitative results provided solid evidence on the long blood circulation characteristics and tumour accumulation of $m$-NC over $24 \mathrm{~h}$.

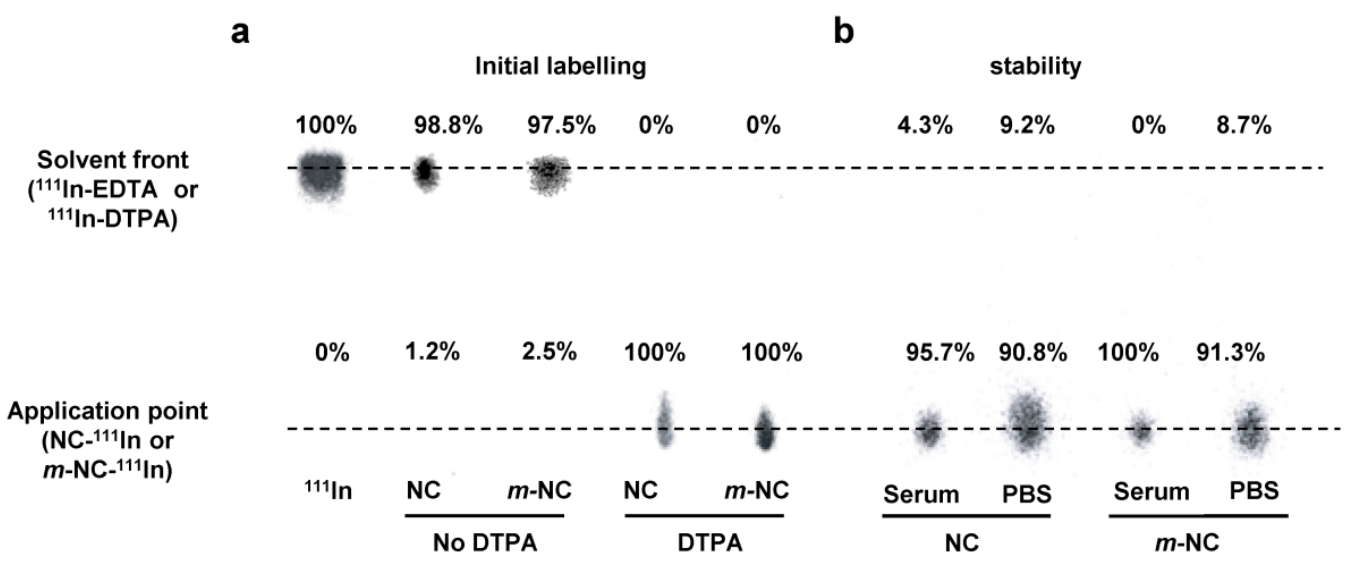

Figure 3. Radio-labelling and in vitro serum stability of NC and $\boldsymbol{m}-\mathbf{N C}$. The NC and $m$-NC were prepared by single emulsification/solvent evaporation method. $\mathrm{PLGA}_{18 \mathrm{KDa}}-\mathrm{PEG}_{3.5 \mathrm{KDa}}-\mathrm{DTPA}$ was incorporated in the formulation at $10 \%(\mathrm{w} / \mathrm{w})$. The radio-labelling reaction was carried out for $30 \mathrm{~min}$ in $0.2 \mathrm{M}$ ammonium acetate $(\mathrm{pH} 5.5)$ and quenched by the addition of 0.1 M EDTA of $1 / 20(\mathrm{v} / \mathrm{v})$ of the total volume. a, Radio-labelling efficiency was evaluated immediately after labelling and $100 \%$ radio-labelling efficiency was obtained for both types of NCs whereas NCs without incorporation of DTPA showed no radio-labelling. $\mathbf{b}$, Serum stability was tested by incubation of NC-IIIln and $m$-NC- IIIIn in serum or PBS at $37^{\circ} \mathrm{C}$ up to $24 \mathrm{~h}$. Both types of NC exhibited high stability in serum and PBS indicated by presence of low amount of IIIIn-EDTA. 
To confirm the magnetic targeting by nuclear imaging, the same experiment above was repeated except that a magnet was applied on one tumour. SPECT/CT imaging and gamma scintigraphy were conducted at 1, 4 and $24 \mathrm{~h}$ post-injection (Fig. 4). The increase in tumour accumulation of $m$-NC due to the magnet application is shown at $1 \mathrm{~h}$ post-injection (immediately after removal of the magnet) and the enhanced uptake could be still seen at $24 \mathrm{~h}$ post-injection (Fig. 4a). Organ biodistribution profiles
(Fig. 4b), other than tumour, were similar to that obtained without application magnetic field (Fig. S4d). Quantitative gamma scintigraphy studies (Fig. 4c) showed that the application of magnetic field increased $m$-NC uptake in $m$-TU from $0.87 \pm 0.09 \%$ $\mathrm{ID} / \mathrm{g}$ to $2.43 \pm 0.27 \% \mathrm{ID} / \mathrm{g}$ at $1 \mathrm{~h}$ post-injection $(\mathrm{p}<0.01)$ and from $2.92 \pm 0.19 \%$ to $5.96 \pm 0.26 \% \mathrm{ID} / \mathrm{g}$ at $24 \mathrm{~h}$ post-injection $(\mathrm{p}<0.01)$. The results suggested $\sim 3.0$ - and $\sim 2.2$-fold increases in tumour uptake at $1 \mathrm{~h}$ and $24 \mathrm{~h}$, respectively (Fig. 4 d). a

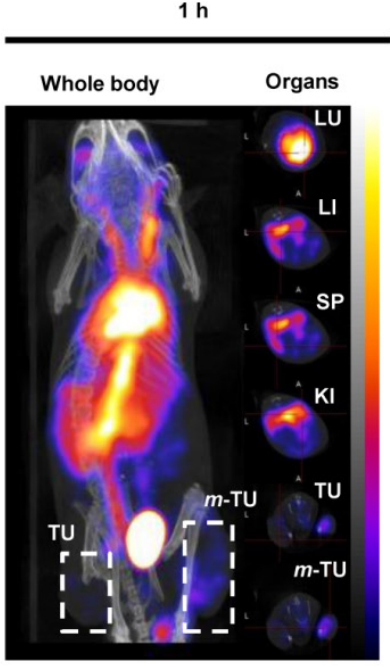

b

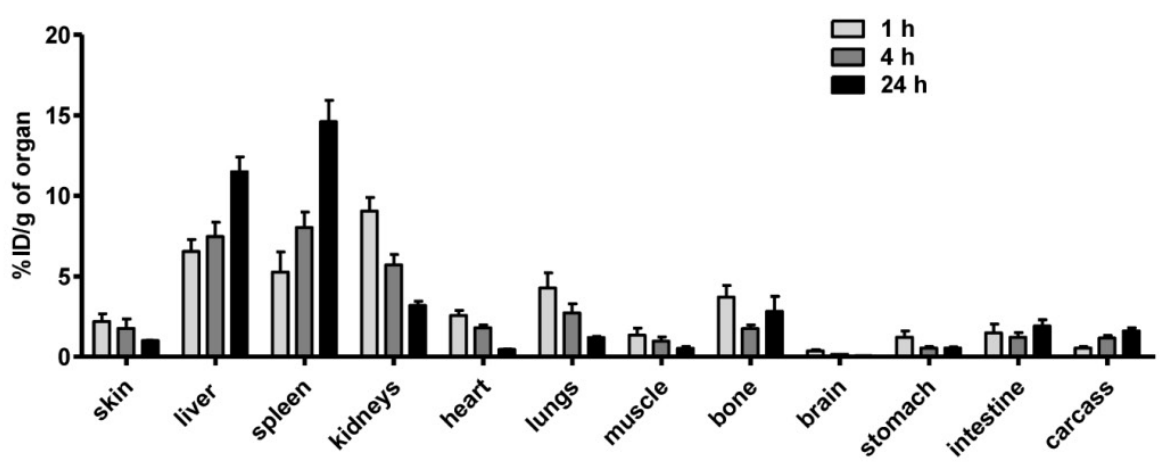

C

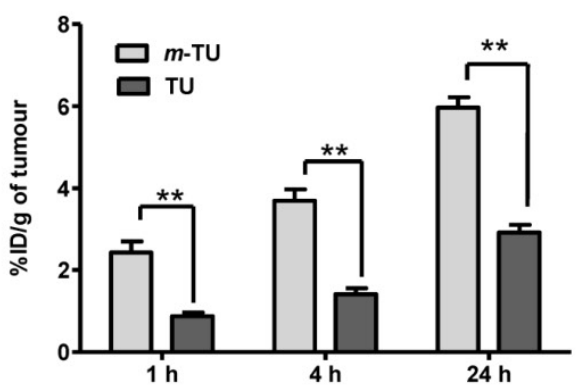

$4 \mathrm{~h}$

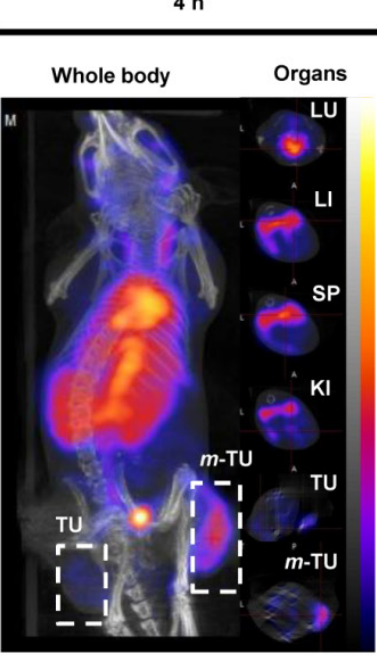

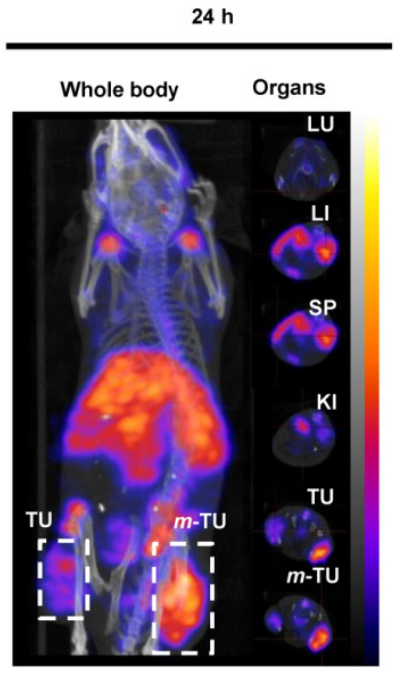

d

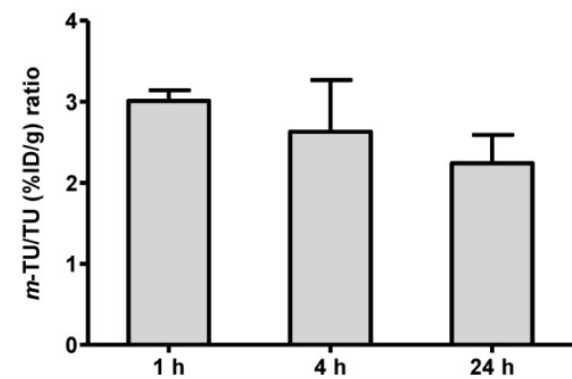

Figure 4. In vivo SPECT/CT imaging and organ biodistribution studies of $\mathbf{m}$-NC-111/n in CT26 tumour-bearing balb/c mice under the influence of magnetic field. Mice were iv injected with $\mathrm{m}$-NC-111/n (8-10 MBq per mouse). A permanent magnet (0.515 T) was applied at one tumour site ( $\mathrm{m}$-TU) for $1 \mathrm{~h}$. Organs were excised at 1 , 4 and $24 \mathrm{~h}$ post-injection for gamma counting. a, Whole body 3D SPECT/CT imaging at 1, 4 and 24 h post-injection. Cross-sections were from lung (LU), liver (LI), spleen (SP), kidney $(\mathrm{KI})$, non-magnetically targeted tumour (TU) and magnetically targeted tumour $(m-\mathrm{TU})$ at equivalent time points; Tumours are marked in dashed square; b, Organ biodistribution profile with values expressed as \% ID/g of organ. c, Quantification of \% ID/g of tumour in $m$-TU and TU at different time points; $d$, Fold increase in \% ID/g of tumour (\%ID/g $m$-TU/\%ID/g TU) upon application of magnetic field. Significant increase in tumour uptake was shown upon application of a magnet at all time points (**p < 0.01$)$. Results are expressed as mean \pm S.D. $(n=3)$. 
Animals were also housed in metabolic cages for urine and faeces collection up to $24 \mathrm{~h}$ to assess excretion profile. Relatively high amount of radioactivity (37.57 $\pm 15.41 \%$ ID/mouse) was eliminated into urine within $24 \mathrm{~h}$ (Fig. S4c). No ${ }^{111}$ In-DTPA/EDTA chelates were detected in the urine samples by TLC, therefore indicating the presence of ${ }^{111} \mathrm{In}$-labelled polymers in urine due to disassembly of $m$-NC-111In (data not shown). Unlike the high radio-activity detected in urine sample, negligible values (< $1 \%$ ID/mouse) were detected in faeces within $24 \mathrm{~h}$, suggesting limited biliary excretion.

Some studies questioned if the apparent targeting effect is due to an increased amount of magnetised nanoparticles within the tumour vasculature under the influence of magnetic force. $\left.{ }^{[29}, 30\right]$ Since image-based results cannot fully discriminate the presence of $m$-NC in tumour vasculature or tumour mass, the distribution of $m$-NC in tumour tissues was further investigated after whole body saline perfusion prior sacrificing the animals (Additional file 1: Fig. S5). Perfusion resulted in non-significant reductions $(\mathrm{p}>0.05)$ in non-magnetic tumour uptake from $0.87 \pm$ $0.09 \%$ and $2.92 \pm 0.19 \%$ to $0.79 \pm 0.03 \%$ and $2.55 \pm$ $0.19 \% \mathrm{ID} / \mathrm{g}$ after 1 and $24 \mathrm{~h}$, respectively (Fig. S5b). Interestingly, no reduction in tumour accumulation at the magnetically-targeted tumour site occurred after perfusion at all time points, which confirmed that $m$-NC was localized in the tumour mass and not stuck in tumour vasculature. In addition, the different tumour uptake profiles of $m$-TU and TU obtained with or without perfusion also suggested that the application of magnetic field not only helped the accumulation of $m$-NC at tumour site but the $m$-NC was capable of extravasation into tumour mass.

\section{In vivo $M R$ imaging}

Owing to the intrinsic MR contrast properties of SPION, the $m$-NC distribution in tumour was further monitored by in vivo MR imaging, an imaging technique providing high resolution images and excellent soft tissue contrast. The tracking of the biodistribution of encapsulated SPIONs upon application of magnetic field was also indirect evidence on the stability of SPION in $m$-NC in vivo. The MR contrast properties of SPION and $m$-NC were evaluated in vitro using $1 \%$ agarose phantom $\left(\mathrm{T}_{2}=15 \mathrm{~ms}\right.$, Fig. $\left.5 \mathbf{a}\right)$. The transverse $\left(T_{2}\right)$ relaxation times were measured at different concentrations and $R_{2}$ values were calculated. Interestingly, $m$-NC exhibited higher $\mathrm{r}_{2}$ relaxivity $\left(404 \mathrm{mMs}^{-1}\right)$ compared to the non-encapsulated SPION (260 $\mathrm{mMs}^{-1}$ ) (Fig. 5b) and the commercial Feridex I.V.® (224 $\mathrm{mMs}^{-1}$ reported in the literature) [11]. The high $\mathrm{r}_{2}$ relaxivity is likely due to the clustering of SPION in $m$-NC, which was shown in the cryo-EM images, thereby enhancing the local magnetic field.[31-33] A higher molar relaxivity is important especially for applications such as tumour MR imaging due to the low sensitivity of MRI and low tumour accumulation of contrast agents compared to RES organs i.e. liver and spleen, when conventional MRI contrast agents are used.

In vivo $\mathrm{MR}$ imaging was performed at $m-\mathrm{NC}$ pre-injection and at 1, 4, $24 \mathrm{~h}$ post injection of $m$-NC. The representative coronal $\mathrm{T}_{2}$-weighted images at an acquired echo time of $15 \mathrm{~ms}$ are shown (Fig. 5c) and the $R_{2}$ relaxation rates of tumours and adjacent muscles at different post-injection time are shown in Additional file 1: Fig. S6. As expected, the $\mathrm{R}_{2}$ relaxation rates of the tumour-adjacent muscle tissues reported similar values to that of pre-injected muscles at any post-injection time point (Fig. S6a). In contrast, pre-injected tumours appeared brighter than muscles and both $m$-TU and TU were darkened after injection (Fig. 5c, dashed lines). Such lack of MRI signals corresponded to a shortening in $R_{2}$ values (Fig. $\mathbf{S 6 b}$ ). The tumour accumulation and magnetic targeting effect of $m$-NC was expressed as time-course percentage changes in transverse relaxation rate $\left(d \Delta R_{2}\right)$ over the whole tumour area (Fig. 5c, dashed lines). After administration of $m-\mathrm{NC}$, a significant increase in $\mathrm{d} \Delta \mathrm{R}_{2}$ $(40.24 \pm 4.61 \%)$ was seen in the non-magnetically targeted tumours at $1 \mathrm{~h}$ post-injection and the percentage increased to $57.0 \pm 03.87$ and $64.04 \pm 4.34 \%$ at 4 and 24 $\mathrm{h}$, respectively, as a result of passive accumulation of $m$-NC (Fig. 5d). Compared to the non-magnetically targeted site, more significant darkness was observed in $m$-TU upon application of magnetic field, with $\mathrm{d} \Delta \mathrm{R}_{2}$ increased to $80.07 \pm 7.23 \%(\mathrm{p}<0.05), 91.55 \pm$ $6.65 \%(\mathrm{p}<0.05)$ and $97.71 \pm 5.55 \%(\mathrm{p}<0.01)$ at 1,4 and $24 \mathrm{~h}$ (Fig. 5d), respectively, representing a 2.09-, 1.57and 1.51-fold increase compared to the non-targeted tumour (Fig. 5e), respectively. The decreasing tendency in $d \Delta R_{2}$ fold increase could be attributed to the EPR effect over time and these results were in line with the data obtained from gamma scintigraphy studies. In our studies, the $\mathrm{T}_{2}$ hypointensity (darkening) caused by the high SPION accumulation was observed at $m$-TU as early as $1 \mathrm{~h}$ post-injection, suggesting that the proposed $m$-NC offered sufficient sensitivity to be monitored in tumours, and also confirmed the success of magnetic targeting in vivo.

In most previous magnetic targeting studies, the magnetic targeting effect was investigated by MR imaging only as SPION is MR active and drugs were either chemically conjugated or physically absorbed in the coating matrix of magnetic nanoparticles (MNP)/ferrofluid. [34-41] In this work, a multi-functional $m$-NC was developed to facilitate magnetic targeting to tumour. Nanocapsules contain an 
oily core to solubilise and encapsulate a relatively large quantity of hydrophobic molecules, thus can serve as drug reservoirs with sustained release of anti-cancer agents.[25] The $m$-NC was also capable of incorporating with three different imaging moieties, i.e. NIR dye, SPION and radio-isotope chelator, as a multi-modal imaging probe. The time-course organ distribution and tumour retention of the magnetic nanocarrier as a function of magnetic field could therefore be monitored with triple-modal fluorescence/MR/SPECT imaging modalities. Optical imaging is known to be limited by the depth of light penetration due to its scattering and tissue absorption [42]. It is therefore considered to be a semi-quantitative imaging tool. However, the short acquisition time and low-cost of optical labelling methods make it a fast-screening and lead selection tool in cancer theranostics. Contrary to optical imaging, nuclear imaging permits accurate and sensitive quantification of $m$-NC biodistribution. Organ distribution, blood circulation, excretion profiles, tumour accumulation and magnetic targeting efficacy of $m$-NC were obtained from SPECT/CT imaging and gamma scintigraphy. a

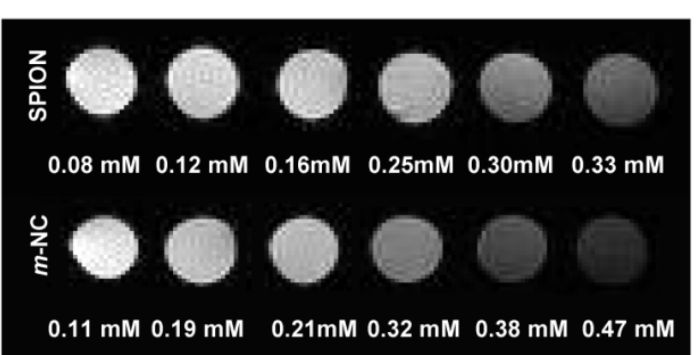

b

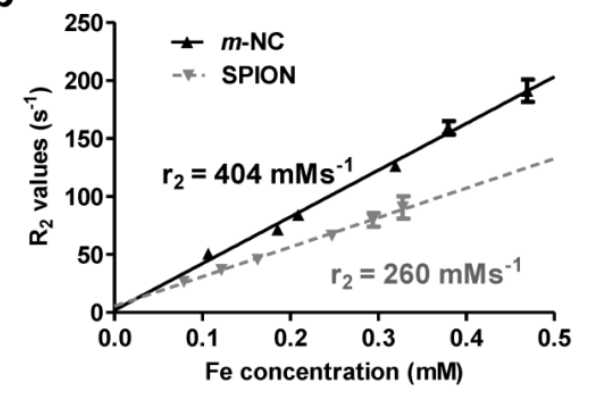

C
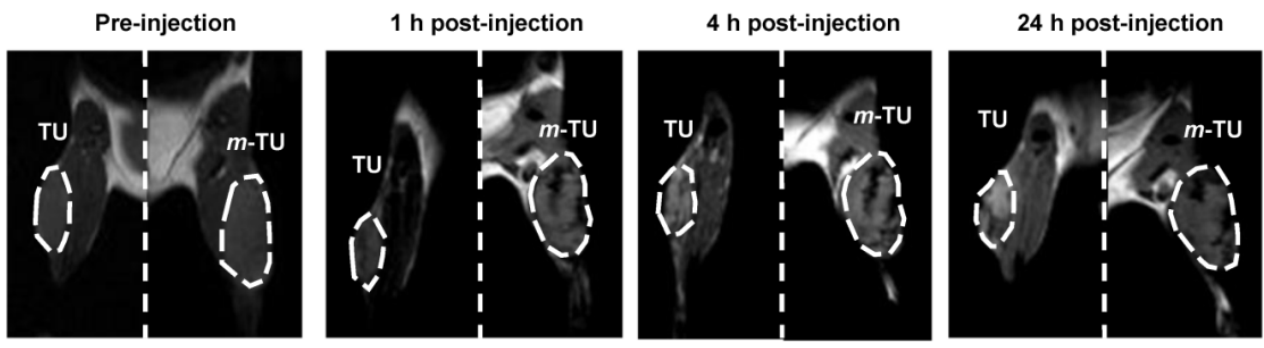

d

e
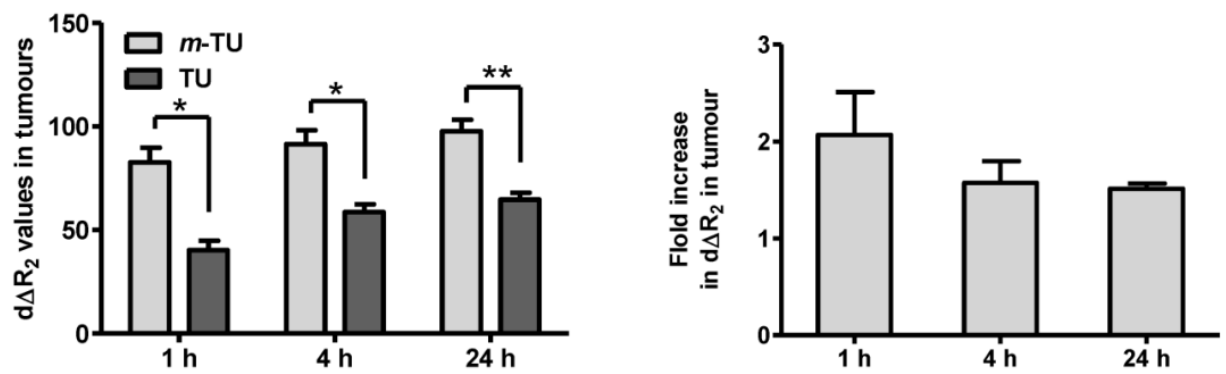

Figure 5. Phantom and in vivo $\mathrm{T}_{2}$-weighted MR studies of $\boldsymbol{m}$-NC in CT26 tumour-bearing balb/c mice under the influence of magnetic field. Phantom samples of SPION and $m-N C$ were prepared in $1 \%$ agarose. Images were obtained using a 7 Tesla $(7 \mathrm{~T})$ preclinical MR scanner. a, Phantom MR imaging of $m-\mathrm{NC}$ and SPION. b, R2 relaxation rate analysis as a function of Fe concentration. For in vivo MR study, mice were i.v injected with $m-\mathrm{NC}$ at a dose of $125 \mathrm{mg}$ SPION/Kg. A permanent magnet $(0.515 \mathrm{~T})$ was applied at one tumour site ( $m$-TU) for $1 \mathrm{~h}$. c, MR images of tumours at $0,1,4$ and $24 \mathrm{~h}$ post-injection. Tumours are marked in dashed lines. d, Percentage changes in transverse relaxation rates $\left(d \Delta R_{2}\right)$ in magnetically targeted tumour $(m-T U)$ and non-magnetically targeted tumour (TU) over time. e, Fold increase in $d \Delta R_{2}$ in $m$-TU compared to $T U$ as a function of time; Significant increase in tumour uptake was shown upon application of a magnet at all time points $\left({ }^{*} p<0.05\right.$, $\left.* * p<0.01\right)$. Results are expressed as mean \pm SD $(n=$ 3). 
In addition to the whole body biodistribution, the high spatial resolution MR images provided the regional distribution profiles of $m$-NC in tumours. The fold increase in $m$-TU accumulation obtained by MRI was slightly lower than the values offered by nuclear imaging. This is likely due to lower sensitivity of MRI compared to SPECT. Weak interactions between MRI static magnetic field and body tissues result in high signal-to-noise ratio and limit MRI sensitivity to $10^{-3}-10^{-5} \mathrm{~mol} / \mathrm{L}$, whereas nuclear imaging has a significantly higher sensitivity of $10^{-10} 1^{-12}$ $\mathrm{mol} / \mathrm{L}$.[43] As a result, the latter is more accurate and reliable for quantification. In this work, similar trend of magnetic targeting efficacy over time was observed in tumours with both techniques. The highest fold increase in $m$-TU uptake was seen at $1 \mathrm{~h}$ post-injection, immediately after the magnet removal. The reduction at 4 and $24 \mathrm{~h}$ was a result of the EPR effect taking place in both TU and $m$-TU over time. The good correlation between information obtained from MRI (SPION) and SPECT (polymeric shell), however, suggests that most of the SPION was stably encapsulated and remained in the $m$-NC when magnetically targeted to tumours. On the other hand, MRI provides high spatial resolution up to 50-250 $\mu \mathrm{m}$ while SPECT imaging is limited to only $0.3-1 \mathrm{~mm}$ [43] The hypointense signals in MR images from $m$-NC were shown in tumours in a heterogeneous manner and this could be due to the regional differences in tumour growth rates, vascular densities, vascular permeability and presence of other tumour-associated cell types.[17] The heterogeneous distribution of $m$-NC in tumours was likely to be a result of an inhomogeneous tumour blood vessel pattern, therefore the hypointense regions might correlate to areas of high vascularity.[33]

At the current stage, magnetic targeting is considered for loco-regional treatment of cancers that are close to body surface, i.e, advanced head and neck tumours, skin tumours and breast cancer.[44, 45] Attenuated gradients pose a translational challenge for target sites located in deep tissues, as magnetic flux density wears off dramatically with distance.[46, 47] To overcome these barriers, a number of studies have been proposed to generate localised magnetic field using implanted magnets near the targets. [48, 49] Alternatively, magnetic flux density can be focused from a distance through the use of custom designed magnetic devices. The latter approach is currently under investigations, for magnetic cell delivery, and is claimed to be suitable for deep tissue magnetic capture.[50]

\section{Histological examination}

Cytotoxicity of four types of NCs, namely
non-PEGylated $\mathrm{NC} / m$-NC and PEGylated $\mathrm{NC} / m$-NCs was assessed in CT26 cells in vitro by MTT assay. As expected, all types of $\mathrm{NC}$ formulations showed no significant reduction in cell viability, compared to naive group, over 72 hours at maximum polymer concentration that might accumulate in tumour in vivo (Additional file 1: Fig. S7). Histological examination of fixed and stained tissue sections was performed at $24 \mathrm{~h}$ post-injection. H\&E staining showed no major signs of necrosis in heart lung, liver, spleen and kidneys of mice injected with $\mathrm{NC}$ and $m$-NC at 24 h post-injection (Fig. 6). No extended tumour necrosis was seen in all treated tumours with or without magnetic field in NC and $m$-NC injected mice compared to control. Mice injected with $m$-NC showed brownish spots seen evenly distributed in liver, spleen and some areas of the magnetically targeted tumour, indicating the presence of SPION (Fig. 6, red arrows).

Perl's staining was employed to confirm the presence of iron in tissues (Fig. 6, black arrows). No positive signs of iron were seen in control organs except for spleen where blue spots were seen due to endogenous iron.[51] Very few blue-stained regions were observed in lung of $m$-NC treated mice whereas strongly positive signals were homogenously distributed in liver and spleen, which co-localized with the brownish spots observed in H\&E stained sections (Fig. 6, red arrows). More importantly, a number of positive blue signals appeared in $m$-TU of mice administrated with $m$-NC while only a few spots were seen in TU, indicating the significant increase in $m-\mathrm{NC}$ tumour uptake as a result of magnetic targeting.

\section{Conclusions}

A polymeric nanocapsule formulation for triple-modal fluorescence/MR/SPECT imaging and magnetic targeting was developed. $m$-NC was capable of encapsulating a high amount of SPION, which was crucial to achieve magnetic drug targeting and MRI in vivo. NIR dye (ICG) and radio-isotope chelator (DTPA) were either physically encapsulated or chemically conjugated to the $m$-NC to facilitate optical imaging and nuclear imaging, respectively. Optical imaging was proven to be a fast-screening method to assess the tumour uptake and the long circulation property of $m$-NC whereas SPECT/CT imaging allowed quantitative assessment of organ biodistribution, passive tumour accumulation and magnetic targeting efficacy of $m$-NC. High resolution MR imaging showed heterogeneous distribution patterns of $m$-NC in tumours, indicating that the proposed $m-\mathrm{NC}$ offered sufficient sensitivity to monitor its tumour uptake and magnetic targeting in vivo. Time-course optical/MR/SPECT imaging studies showed enhanced 
tumour uptake upon the application of the external static magnetic field at tumour site. Magnetic targeting led to $\sim 3$ and $\sim 2.2$ fold increase in tumour uptake at 1 and $24 \mathrm{~h}$, respectively compared to only passive

NC

$\begin{array}{ll}\text { NC } & \\ \text { H\&E } & \text { Perl's Stain }\end{array}$
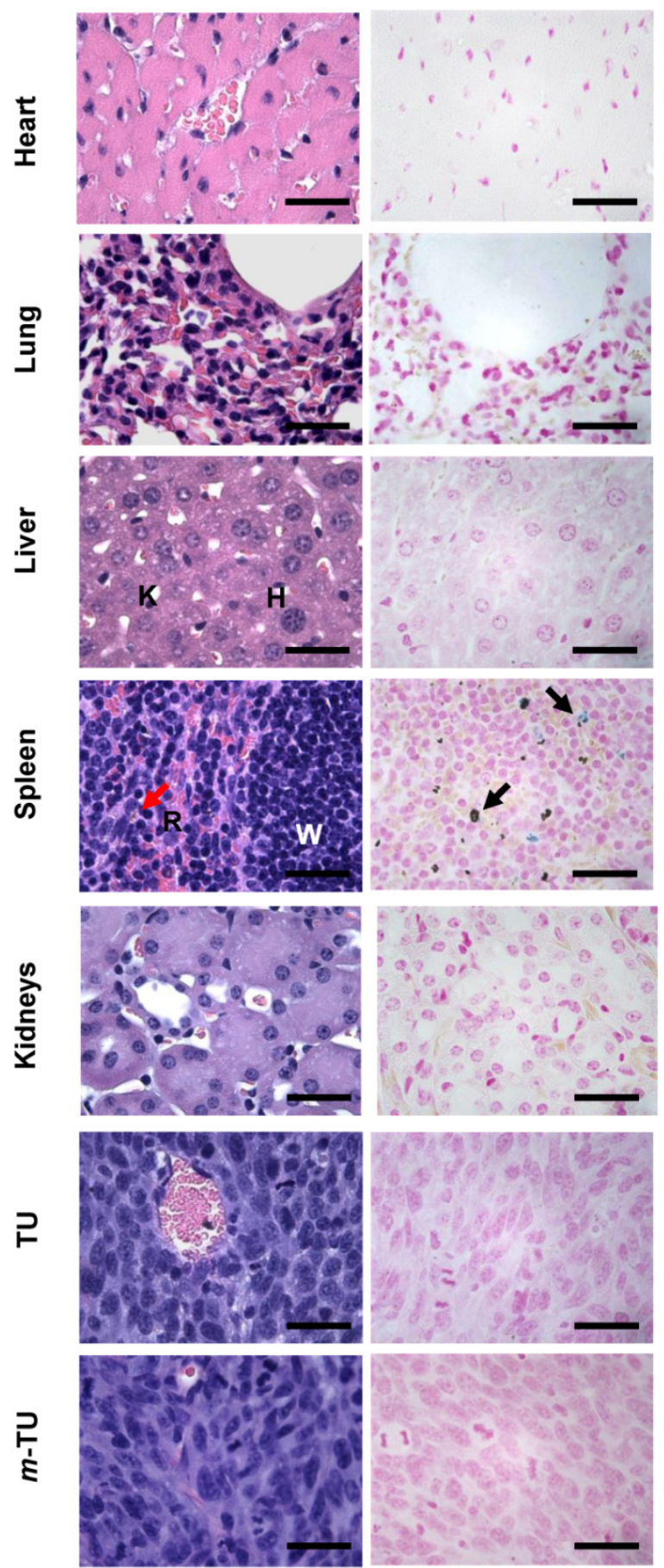

targeting. Findings from this work demonstrated the capability of the $m$-NC to be used as a triple-modal imaging nanocarrier for cancer magnetic targeting in vivo.

$m-\mathrm{NC}$
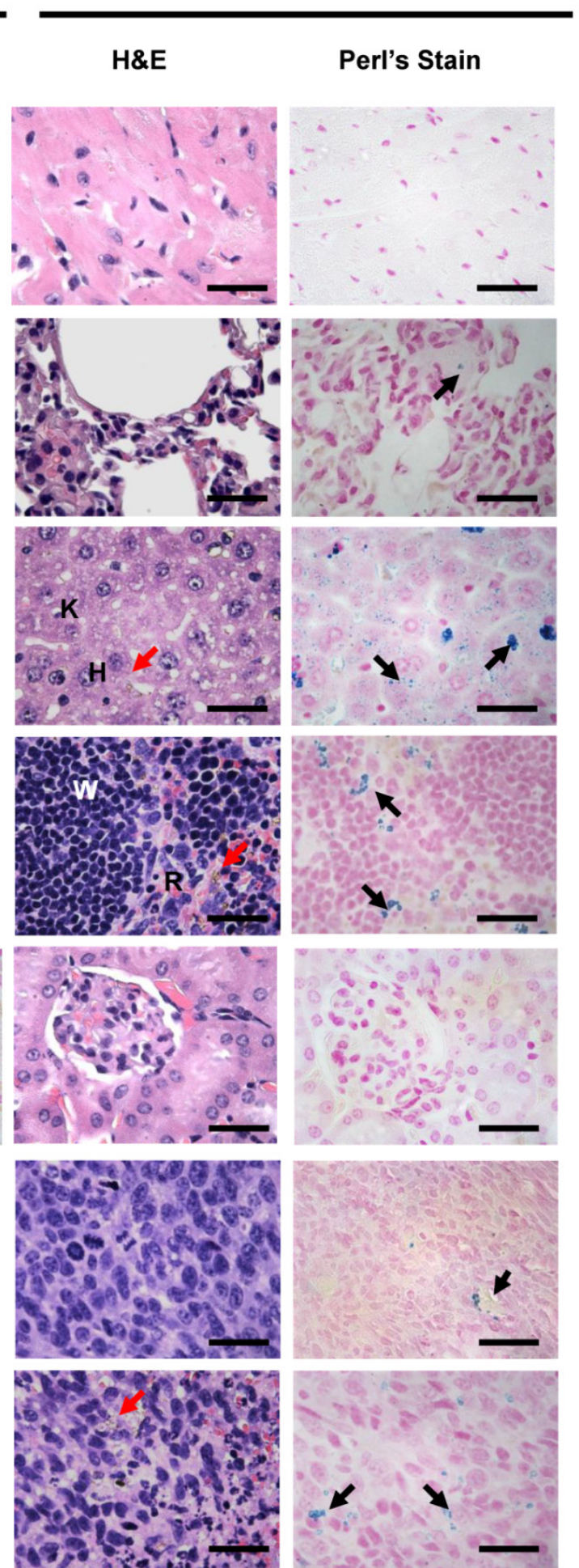

Figure 6. Microscopic examination of lung, liver, spleen and tumour sections of CT26 tumour-bearing balb/c mice. Mice were intravenously injected with NC (left panel) and $m$-NC (right panel) with application of magnet at one tumour site ( $m$-TU). Organs were sampled at $24 \mathrm{~h}$ post-injection, sectioned and stained with H\&E and Perl's staining. Red and black arrows point to the presence of iron in H\&E (brownish spots) and Perl's staining (blue stains), respectively. H, K and W, R stands for hepatocytes, Kupffer cells in liver and white pulp, red pulp in spleen, respectively. Scale bars are $30 \mu \mathrm{m}$. No histological abnormalities were observed in all organs at $24 \mathrm{~h}$ post-injection. Increased amount of blue spots could be seen in the magnetically targeted tumour, indicating the enhancement in $m$-NC tumour uptake by magnetic targeting. 


\section{Materials and methods}

\section{Materials}

SPION (oleic acid coated iron oxide nanoaprticles with diameter of $10 \mathrm{~nm}, 10^{17}$ particles per ml) was purchased from Magnacol Ltd (UK). Soybean lecithin (Epikuron $140 \mathrm{~V}$ ) was a kind gift from Cargill Pharmaceuticals (USA). Polyoxyethylene-bis-amine $\left(\mathrm{NH}_{2}-\mathrm{PEG}_{3.5 \mathrm{KDa}}-\mathrm{NH}_{2}\right)$ was purchased from JENKEM (USA). D/L-lactide/glycolide copolymer 75/25 (PLGA $18 \mathrm{KDa}-\mathrm{COOH}$ ) was purchased from Purac Biomaterials (Netherlands). Tween ${ }^{\circledR} 80$ was obtained from Fisher Scientific Ltd (UK). Sodium chloride, phosphate buffered saline tablets, dicyclohexylcarbodiimide (DCC), N-Hydroxysuccinimide (NHS), anhydrous dimethylformamide (DMF), diethylene triamine pentaacetic acid (DTPA), castor oil and potassium permanganate were purchased from Sigma Aldrich (UK). Indocyanine green (ICG) was purchased from MP Biomedicals (USA). Dichloromethane (DCM), isopropanol, dimethylsulphoxide (DMSO), triethylamine $\left(\mathrm{Et}_{3} \mathrm{~N}\right)$ and diethyl ether were obtained from Fisher Scientific Ltd (UK). Advanced RPMI-1640 media, penicillin-Streptomycin 100x, Trypsin-EDTA (1X) with Phenol red Glutamax ${ }^{\mathrm{TM}}$ supplement, phosphate buffered saline PBS (10x, pH 7.4) were obtained from Gibco, Invitrogen (UK). Plasma derived Bovine serum (FBS) was obtained from First-Link Ltd (UK). Pentobarbital sodium (Euthatal $\left.{ }^{\circledR}\right)$ was obtained from Merial (UK). SP-Sephadex C25 cation-exchanger column was obtained from Phenomenex Inc (USA). C18 Sep-Pak ${ }^{\circledR}$ Vac column was purchased from Waters Ltd (USA). Dialysis tubing SnakeSkin (MWCO $10 \mathrm{KDa}$ ), black 96 well-plates and autoclave tapes were purchased from Thermo-fisher Ltd. (USA). Dialysis tubing (MWCO $2 \mathrm{KDa}$ ) was obtained from Sigma Aldrich (UK). Disposable square polystyrene cuvettes and disposable capillary cells were purchased from Malvern Instrument (UK). Mica sheets $(11 \mathrm{~mm} \times 11 \mathrm{~mm} \times 0.15 \mathrm{~mm})$ were used for atomic force microscopy experiments (AFM). PD-10 desalting column was obtained from GE Healthcare Life Sciences (UK). All the reagents were analytical grade and used without further purification.

\section{Formulation of the $\boldsymbol{m}$-NCs}

$\mathrm{PLGA}_{18 \mathrm{KDa}}-\mathrm{PEG}_{3.5 \mathrm{KDa}}-\mathrm{NH}_{2}$

and

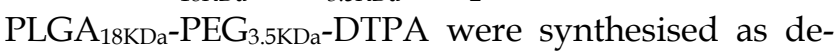
scribed in supplementary method. The PEGylated $m$-NC was prepared by single emulsification/solvent evaporation method. Briefly, polymer $(12.5 \mathrm{mg})$, castor oil $(75 \mathrm{mg})$, soybean lecithin $(25 \mathrm{mg})$ and SPIONs $(2.5 \mathrm{mg})$ were dissolved in $2.5 \mathrm{ml}$ dichloromethane. The organic phase was poured into an aqueous phase
$(5 \mathrm{ml})$ containing Tween ${ }^{\circledR} 80(20 \mathrm{mg})$. The resultant dispersion was emulsified by ultra-sonication, followed by organic phase evaporation. The obtained $m$-NC suspension was further condensed by 10 or 20 times with final polymer concentration of 25 (for optical or MR imaging) or $50 \mathrm{mg} / \mathrm{ml}$ (for SPECT/CT).

\section{Size and Zeta measurements}

The hydrodynamic size (Z-Average), polydispersity index (PDI) and Zeta potential of the NC were determined by NanoZS (Malvern Instrument, UK) using disposable square polystyrene cuvettes (Malvern Instrument, UK) for size and PDI, or disposable capillary cells (Malvern Instrument, UK) for Zeta potential at $25^{\circ} \mathrm{C}$. For size measurement, NC samples were diluted with deionised water. The Z-Average diameter and polydispersity index were presented as the average value of three measurements, with 15 runs within each measurement. Electrophoretic mobility was used to calculate the Zeta potential measurement. NC samples were diluted with deionised water. Three measurements were performed with 20-25 runs within each measurement. The mean and standard deviation of size and Zeta potential were calculated for each sample.

\section{Atomic force microscopy (AFM) of $\boldsymbol{m}$-NC}

The surfaces for AFM analysis were prepared as follows: The different NC formulations were filtrated through a PD-10 desalting column and solutions of 50 $\mu \mathrm{g} / \mathrm{ml}$ were prepared. NC suspensions $(500 \mu \mathrm{l})$ were deposited on a new piece of mica and lyophilised using a Lytrap freeze-dryer (LTE Scientific, UK). Tapping mode AFM analysis (TM-AFM) on the mica substrates was carried out in air at $25{ }^{\circ} \mathrm{C}$ using a Bruker Dimension ICON with Scan Assist. The surfaces were imaged with a general purpose tapping tip made by MikroMasch in Estonia (NSC15/no Al, tip radius $<10 \mathrm{~nm}$; tip height $=20-25 \mu \mathrm{m}$; cone angle $<40$; cantilever thickness $=3.5-4.5 \mu \mathrm{m}$; cantilever width $=$ $32-28 \mu \mathrm{m}$; cantilever length $=120-130 \mu \mathrm{m}$; frequency $f_{0}=265-400 \mathrm{kHz}$; force constant $\mathrm{k}=20-75 \mathrm{~N} \mathrm{~m}^{-1}$, VEECO, USA). The statistical analysis of the AFM images was carried out using WSxM v5.0 software (Spain).

\section{Cryo Transmission Electron Microscopy (Cryo-TEM) of $m$-NC}

The shape, morphology and size distribution of the nanocapsules was evaluated using low-dose cryo-transmission electron microscopy (cryo-TEM) and cryo electron tomography. Cryo-TEM enables the investigation of colloidal solutions close to their native state. A drop of solution $(3 \mu \mathrm{L})$ was applied on hydrophilic TEM Quantifoil grids. The grids were then blotted for $2 \mathrm{sec}$ and plunged into the liquid 
ethane pool using a FEI Vitrobot mark IV, in order to produce a thin vitreous ice layer with embedded assemblies in the holes of the grid. Digital cryo-TEM images were acquired using a FEI Tecnai Spirit operated at $120 \mathrm{kV}$ using a Gatan 626 cryo-transfer tomography holder. The tomographic series was acquired using automated acquisition software Xplore3D from FEI. Next, the series was aligned and reconstructed using the simultaneous iterative reconstruction technique (SIRT) based on 30 iterations carried out using the FEI inspect 3D tomography package. The measurements of size distribution were carried out in Amira (3D visualisation software).

\section{Thermogravimetric Analysis (TGA) of SPION and $\boldsymbol{m}$-NC}

The amount of oleic acid coating on the SPION and the weight percentage of SPION in $m$-NC were characterised by the thermogravimetric analysis (TGA) using TGA Q500 (TA instrument), where about $10 \mathrm{mg}$ of SPION was loaded into platinum pan. The measurement was pre-equilibrated at $100{ }^{\circ} \mathrm{C}$ and then heated from $100{ }^{\circ} \mathrm{C}$ to $1000^{\circ} \mathrm{C}$ with a temperature ramp of $10^{\circ} \mathrm{C} / \mathrm{min}$ under compressed air atmosphere with balance and sample purge flow at 10 and 90 $\mathrm{ml} / \mathrm{min}$, respectively.

\section{Encapsulation efficiency of SPION in $\boldsymbol{m}$-NC}

The SPION encapsulation efficiency was determined by inductively couple plasma mass spectrometry (ICP-MS). $m$-NCs with $1.85 \% \mathrm{w} / \mathrm{w}$ SPION $/ m$-NC (corresponding to $20 \% \mathrm{w} / \mathrm{w}$ SPION/polymer) were prepared and purified by size exclusion chromatography (G75 column) to remove any un-encapsulated SPION. For ICP-MS measurements, suitable dilutions of Fe standards (Leeman Labs Inc., MA) were prepared in $20 \%$ nitric acid to obtain a standard curve in the range of 10-10000 parts per billion with respect to Fe. $m$-NC samples were digested in $2 \mathrm{ml}$ of nitric acid and incubated overnight at $50{ }^{\circ} \mathrm{C}$. The resulting solution was diluted to reach concentration calibration range. The Fe content was determined by inductively couple plasma mass spectrometry (Perkin Elmer SCIEX ICP mass spectrometer, ELAN DRC 6100, USA).

\section{Radio-labelling of NCs and serum stability studies}

The radioactive probe ${ }^{111} \mathrm{InCl}_{3}$ was purchased from Mallinckrodt Pharmaceuticals (The Netherlands) as an aqueous solution in $0.5 \mathrm{M} \mathrm{HCl}$ and used without further purification. To radio-label the NC and $m$-NC with indium-111, NCs were prepared as described in the previous section except that PLGA $_{18 \mathrm{KDa}}-\mathrm{PEG}_{3.5 \mathrm{KDa}}-\mathrm{DTPA}$ was included at $10 \%$ $(\mathrm{w} / \mathrm{w})$ of total polymer content. The NC suspension $(150 \mu \mathrm{l}, 50 \mathrm{mg} / \mathrm{ml}$ of polymer) was incubated with 2 $\mathrm{M}$ ammonium acetate (one ninth of the reaction volume, $\mathrm{pH} 5.5)$, to which $10 \mathrm{MBq}$ or $1 \mathrm{MBq}$ as ${ }^{111} \mathrm{InCl}_{3}$ was added per injection dose for SPECT/CT imaging and gamma scintigraphy, respectively. The reaction was kept at room temperature for $30 \mathrm{~min}$ with intermittent vortexing every $10 \mathrm{~min}$. Upon completion, the radio-labelling reaction was quenched by the addition of $0.1 \mathrm{M}$ EDTA chelating solution (one twentieth of the reaction volume). ${ }^{111} \mathrm{InCl}_{3}$ alone was also subjected to the same conditions of the labelling reaction as a control.

To evaluate the radio-labelling efficiency, the labelled NC-111In and $m$-NC-111In were spotted on the TLC strips which were developed in $0.1 \mathrm{M}$ ammonium acetate containing $50 \mathrm{mM}$ EDTA as a mobile phase. Strips were allowed to dry before being developed and counted quantitatively using a cyclone phosphor detector (Packard Biosciences, UK). The NC-111In and $m$-NC-111In were passed through PD-10 column before injecting into animals to exchange the ammonium acetate buffer ( $\mathrm{pH}$ 5.5) with PBS (pH 7.4) and to ensure that no free ${ }^{111}$ In-EDTA was present in the injected dose. The NC-111In and m-NC-111In ( 150 $\mu \mathrm{L}$ per injection dose, $25 \mathrm{mg} / \mathrm{ml}$ of polymer) were collected from the column and the radio-labelling efficiency was examined immediately after collection from the column.

To determine the stability of the radio-labelled NC-111In and $m$-NC-111In, $5 \mu \mathrm{L}$ of each sample was incubated with serum $(5 \mu \mathrm{L})$ and PBS $(5 \mu \mathrm{L})$ at $37^{\circ} \mathrm{C}$. At $24 \mathrm{~h}, 1 \mu \mathrm{L}$ of the incubated samples were spotted to the TLC strips and which were developed, and quantified as described above. Free ${ }^{111}$ In-EDTA was detected at the solvent front while radio-labelled NCs were retained at the application point.

\section{Animal studies and tumour inoculation}

All animal experiments were performed in compliance with the UK Home Office (1989) Code of Practice for the housing and care of Animals used in Scientific Procedures. The CT26 murine colon carcinoma (CT26, ATCC $\AA$, CRL-2638 ${ }^{\mathrm{TM}}$ ) were cultured in Advanced RPMI (Roswell Park Memorial Institute) 1640 medium supplemented with 1\% L-glutamine, 1\% penicillin-streptomycin and $10 \%$ fetal bovine serum (FBS) with $5 \% \mathrm{CO}_{2}$ and $95 \%$ air at $37{ }^{\circ} \mathrm{C}$. The harvested CT26 cells were suspended in PBS solution (pH 7.4) and injected subcutaneously and bifocally in the hind foot of the female Balb/c mice aged 4-6 weeks (Harlan, UK) at a concentration of $1 \times 10^{6}$ cells in $20 \mu 1$. After inoculation, the tumour volume was measured on day 7 and then every other day using a digital caliper and calculated using Equation (1): 
Tumour volume $\left(\mathrm{mm}^{3}\right)=(4 / 3) * \Pi(\mathrm{A} / 2)^{2} *(\mathrm{~B} / 2)$ $=0.52 \mathrm{~A}^{2} \mathrm{~B}$

where $A$ and $B$ represent the width and the length of the tumours, respectively.[52]

All experiment was carried out when the tumour volume reached approximately $500 \mathrm{~mm}^{3}$ at approximately about $11-12$ days post-injection.

\section{In vivo optical imaging studies}

Optical imaging was performed using an IVIS $®$ Lumina series III In Vivo Imaging Device (Caliper Life Sciences, Perkin Elmer, USA). CT26 tumour-bearing mice were injected intravenously at a dose of $312.5 \mathrm{mg}$

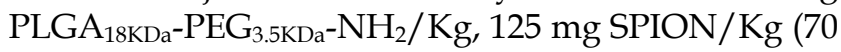
$\mathrm{mg} \mathrm{Fe} / \mathrm{Kg}$ ) and $250 \mu \mathrm{g} \mathrm{ICG/Kg}$. Images of mice were captured for the same animals at 1, 4 and $24 \mathrm{~h}$ post-injection. At $24 \mathrm{~h}$ post-injection, major organs, i.e. heart, lung, liver, spleen, kidneys, intestine and tumours were excised and imaged on a black surface (Caliper Life Sciences, Perkin Elmer, USA). Fluorescence images was obtained using ICG filter $(680,700$, $720,740,760,780 / 845 \mathrm{~nm}$ for excitation/emission wavelengths) with exposure time of $8 \mathrm{~s}$. The collected fluorescence emission signals were stored in efficiency units.

\section{Live small animal SPECT/CT imaging studies}

$m$-NC was radio-labelled with indium-111 as described in previous sections. Whole body imaging of mice injected with $m$-NC-111In was carried out using SPECT/CT imaging. CT26 tumour-bearing Balb/c mice were injected intravenously via a lateral tail vein with $150 \mu \mathrm{l}$ PBS solution containing 8-10 MBq $m$-NC-111 In (corresponding to $312.5 \mathrm{mg}$ polymer $/ \mathrm{Kg}$ and $125 \mathrm{mg}$ SPION/Kg or $70 \mathrm{mg} \mathrm{Fe} / \mathrm{Kg}$ ). The imaging was carried out at 0-30 $\mathrm{min}, 4 \mathrm{~h}$ and $24 \mathrm{~h}$ post-injection under anaesthesia of $1.5 \%$ isoflurane/98.5\% oxygen using Nano-SPECT/CT scanner (Bioscan, Washington DC, USA). SPECT acquisitions were obtained using 16 projections over 40-60 min using a four-head scanner with $1.4 \mathrm{~mm}$ pinhole collimators. CT scans were carried out after the SPECT acquisition. SPECT and CT images were analyzed using InVivoScope ${ }^{\mathrm{TM}}$ software (Bioscan, Washington DC, USA).

\section{Quantitative biodistribution studies by gamma scintigraphy}

Organ biodistribution studies of $m$-NC-111In including organ biodistribution, blood circulation and excretion profile were performed in CT26 tumour-bearing balb/c mice using gamma scintigraphy to obtain quantitative data. Mice were randomly divided into four groups of three mice each and assigned as $1 \mathrm{~h}, 4 \mathrm{~h}, 24 \mathrm{~h}$ and control group. Mice were injected intravenously via a lateral tail vein with $150 \mu l$
PBS solution containing $\sim 0.7 \mathrm{MBq} m$-NC-111 In (corresponding to $312.5 \mathrm{mg}$ polymer $/ \mathrm{Kg}$ and $125 \mathrm{mg}$ SPION/Kg or $70 \mathrm{mg} \mathrm{Fe} / \mathrm{Kg})$. Blood sample $(5 \mu \mathrm{l})$ was collected from the tail vein at $2 \mathrm{~min}, 5 \mathrm{~min}, 10 \mathrm{~min}, 30$ min, $1 \mathrm{~h}, 4 \mathrm{~h}$ and $24 \mathrm{~h}$ post-injection. Tumours and other tissues, i.e. skin, liver spleen, heart, lung, muscle, bone, brain, stomach, intestine, tail and carcass were removed, weighed, and the radioactivity was measured by a gamma counter (1280 CompuGamma Universal Gamma Counter, LKB Wallac, Finland), using the appropriate energy windows for ${ }^{111} \mathrm{In}$. Results were expressed as percentage of injected dose per organ (\% ID/organ) and percentage of injected dose per gram organ $(\% \mathrm{ID} / \mathrm{g})$ as means $\pm \mathrm{SD}(\mathrm{n}=3)$.

\section{In vitro and in vivo MR Imaging}

Phantom and in vivo MR imaging were performed at a 7T horizontal MR scanner (Agilent, Palo Alto, CA). The gradient coil had an inner diameter of $12 \mathrm{~cm}$, gradient strength was $1000 \mathrm{mT} / \mathrm{m}(100 \mathrm{G} / \mathrm{cm})$ and rise-time of $120 \mu \mathrm{s}$. A quadrature transmit/receive coil (RAPID Biomedical GmbH, Germany) with an internal diameter of $39 \mathrm{~mm}$ was used. Animals were intravenously injected with $m$-NC at the same dose used for optical imaging and imaged at $0,1,4$ and $24 \mathrm{~h}$ post injection. A spin-echo MRI technique where echo times (TE) were varied, was used to acquire $\mathrm{T}_{2}$-weigheted images both in vitro and in vivo. The spin-echo parameters in phantoms were: FOV = $30 \times 30 \mathrm{~mm}$, matrix size $=96 \times 96$, slice thickness $=1$ $\mathrm{mm}$; number of slice $=1$; 1 average, $\mathrm{TR}=2000 \mathrm{~ms}$; TE $=10,30,50,75,100$ and $125 \mathrm{~ms}$; scan time $\sim 20 \mathrm{~min}$. For in vivo acquisitions FOV was changed to $25 \times 25 \mathrm{~mm}$ and matrix size to $128 \times 128$. A short-axis and a long axis view were used for the in vivo experiment. MR images were analysed using the ImageJ software (NIH, USA). For in vivo studies, $\mathrm{R}_{2}$ values of $m$-TU and TU were obtained from the mean signal intensity in defined regions of interest shown as dashed line (whole tumour area) in MR images. The $\mathrm{R}_{2}$ values of adjacent muscle were used as a reference to the magnetic targeting enhancement $R_{2}$ values. The changes in $\mathrm{R}_{2}$ due to the presence of $m-\mathrm{NC}$ in tumour were expressed as percentage changes of the initial (pre-injection) relaxation rate by Equation (2) for all animals at all time points:

$$
\mathrm{d} \Delta \mathrm{R}_{2}=\left(\mathrm{R}_{2 \text { post }}-\mathrm{R}_{2 \text { pre }}\right) / \mathrm{R}_{2 \text { pre }} \times 100 \%
$$

\section{Magnetic targeting studies in tumour-bearing mice}

Magnetic targeting studies were carried out in the same tumour model at the same dose of $m-\mathrm{NC}$ described in previous sections. An $8 \mathrm{~mm}$ diameter disk neodymium magnet (first4magnet, UK), rated 0.515 Tesla at its face surface, was placed 
non-invasively over the surface of one of the tumours and retained using autoclave tapes. The other site of tumour was used as an internal negative control as no magnet was applied. After $1 \mathrm{~h}$, the magnet was removed from the mice. The procedures of optical, MR and SPECT/CT imaging were carried out as described in previous sections.

\section{Hematoxylin and Eosin (H\&E) stain of tissue sections}

Small pieces $\left(\sim 5 \mathrm{~mm}^{2}\right)$ of major organs i.e. heart, lung, kidney, liver, spleen and tumour tissues, from animals exposed to NCs and magnet, were immediately fixed in 10\% neutral buffered formalin. Samples were then wax-embedded and sectioned for haematoxylin and eosin stains (H\&E) according to standard histological protocols at the Royal Veterinary College, UK.

\section{Perl's staining of tissue sections}

To detect the presence of iron in tissues, Perl's staining was carried out using a Perl's stain kit used according to the manufacturer's instruction. In brief, sections were de-paraffinised and incubated with potassium ferrocyanide solution for 20 min followed by counterstaining with nuclear red solution for 10 min. Sections were then dehydrated using increasing concentrations of ethanol solutions $(50 \%, 70 \%, 90 \%$, and absolute ethanol), cleared by xylene and mounted in DPX (Distrene, Plasticiser, Xylene) mounting media. All stained sections were analysed using a Leica DM 1000 LED Microscope (Leica Microsystems, UK) coupled with CDD digital camera (Qimaging, UK).

\section{Statistical Analysis}

Quantitative data are presented as mean \pm standard deviation (SD). Paired sample $\mathrm{T}$ tests were performed using IBM SPSS version 20. The $t$-value, degrees of freedom and two-tailed significance ( $\mathrm{p}$-value) were determined. When $\mathrm{p}$-values were 0.05 or less, differences were considered statistically significant.

\section{Supplementary Material}

Additional File 1:

Supplementary methods, Supplementary scheme and figures. http://www.thno.org/v06p0342s1.pdf

Additional File 2:

Movie S1. http:/ / www.thno.org/v06p0342s2.mpg

\section{Acknowledgements}

The authors would like to thank Prof Robert Hider (King's College London) for useful discussion on the chemical functionalization of the polymers, $\mathrm{Mr}$ William Luckhurst (King's College London) on the technical help of AFM measurements and Mr Andrew Cakebread (King's College London) on his technical help of ICP-MS measurements. J.B. acknowledges funding from King's-China Scholarship Council (CSC). J.W. and N.R. acknowledge funding from Biotechnology and Biological Sciences Research Council (BB/J008656/1) and Associated International Cancer Research (12-1054). K.T.AJ. acknowledges funding from EU FP7-ITN Marie-Curie Network programme RADDEL (290023). S.B. acknowledges funding from the European Research Council under the 7th Framework Program (FP7), ERC Starting Grant No. 335078 COLOURATOMS, and the Integrated Infrastructure Initiative No. 262348 European Soft Matter Infrastructure, ESMI.

\section{Competing Interests}

The authors have declared that no competing interest exists.

\section{References}

1. Tran N, Webster TJ. Magnetic nanoparticles: biomedical applications and challenges. J Mater Chem. 2010; 20: 8760-7.

2. Arruebo M, Fernández-Pacheco R, Ibarra MR, Santamaría J. Magnetic nanoparticles for drug delivery. Nano today. 2007; 2: 22-32.

3. Jurgons R, Seliger C, Hilpert A, Trahms L, Odenbach S, Alexiou C. Drug loaded magnetic nanoparticles for cancer therapy. J Phys Condens Matter. 2006; 18: S2893-S2902

4. Montagne F, Mondain-Monval O, Pichot C, Mozzanega H, Elasssari A. Preparation and characterization of narrow sized $(\mathrm{o} / \mathrm{w})$ magnetic emulsion. J Magn Magn Mater. 2002; 250: 302-12.

5. Torchilin VP. Drug targeting. Eur J Pharm Sci. 2000; 11: S81-S91.

6. Yang X, Hong H, Grailer JJ, Rowland IJ, Javadi A, Hurley SA, et al. cRGD-functionalized, DOX-conjugated, and ${ }^{64} \mathrm{Cu}$-labeled superparamagnetic iron oxide nanoparticles for targeted anticancer drug delivery and PET/MR imaging. Biomaterials. 2011; 32: 4151-60.

7. Chen K, Li Z-B, Wang H, Cai W, Chen X. Dual-modality optical and positron emission tomography imaging of vascular endothelial growth factor receptor on tumor vasculature using quantum dots. Eur J Nucl Med Mol Imaging. 2008; 35: 2235-44.

8. Lee H-Y, Li Z, Chen K, Hsu AR, Xu C, Xie J, et al. PET/MRI dual-modality tumor imaging using arginine-glycine-aspartic (RGD)-conjugated radiolabeled iron oxide nanoparticles. J Nucl Med. 2008; 49: 1371-9.

9. Torres Martin de Rosales R, Tavaré R, Glaria A, Varma G, Protti A, Blower PJ. 99mTc-bisphosphonate-iron oxide nanoparticle conjugates for dual-modality biomedical imaging. Bioconjug Chem. 2011; 22: 455-65.

10. Madru R, Kjellman P, Olsson F, Wingårdh K, Ingvar C, Ståhlberg F, et al. 99mTc-labeled superparamagnetic iron oxide nanoparticles for multimodality SPECT/MRI of sentinel lymph nodes. J Nucl Med. 2012; 53: 459-63.

11. Misri R, Meier D, Yung AC, Kozlowski P, Häfeli UO. Development and evaluation of a dual-modality (MRI/SPECT) molecular imaging bioprobe. Nanomedicine: NBM. 2012; 8: 1007-16.

12. Torres Martin de Rosales R, Tavaré R, Paul RL, Jauregui-Osoro M, Protti A, Glaria A, et al. Synthesis of ${ }^{64} \mathrm{Cu}(\mathrm{II})$-bis (dithiocarbamatebisphosphonate) and its conjugation with superparamagnetic iron oxide nanoparticles: in vivo evaluation as dual-modality PET-MRI agent. Angew Chem Int Ed Engl. 2011; 50: 5509-13.

13. Lee H, Yu MK, Park S, Moon S, Min JJ, Jeong YY, et al. Thermally cross-linked superparamagnetic iron oxide nanoparticles: synthesis and application as a dual imaging probe for cancer in vivo. J Am Chem Soc. 2007; 129: 12739-45.

14. Medarova Z, Pham W, Kim Y, Dai G, Moore A. In vivo imaging of tumor response to therapy using a dual-modality imaging strategy. Int J Cancer. 2006; 118: 2796-802.

15. Rimkus G, Grüttner C, Bremer-Streck S, Herrmann K, Krumbein I, Reichenbach J, et al. Mvcam-1 specific iron oxide nanoparticles based probes for multimodal imaging purposes. Biomed Tech (Berl). 2012; 57: 77-80.

16. de Barros AB, Tsourkas A, Saboury B, Cardoso VN, Alavi A. Emerging role of radiolabeled nanoparticles as an effective diagnostic technique. EJNMMI Res. 2012: $2 \cdot 39$.

17. Moore A, Medarova Z, Potthast A, Dai G. In vivo targeting of underglycosylated MUC-1 tumor antigen using a multimodal imaging probe. Cancer Res. 2004; 64: 1821-7. 
18. Chen IY, Wu JC. Cardiovascular molecular imaging focus on clinical translation. Circulation. 2011; 123: 425-43.

19. Ding $\mathrm{H}, \mathrm{Wu} F$. Image guided biodistribution and pharmacokinetic studies of theranostics. Theranostics. 2012; 2: 1040-53.

20. Ting G, Chang C-H, Wang H-E. Cancer nanotargeted radiopharmaceuticals for tumor imaging and therapy. Anticancer Res. 2009; 29: 4107-18.

21. Thomas R, Park I-K, Jeong YY. Magnetic iron oxide nanoparticles for multimodal imaging and therapy of cancer. Int J Mol Sci. 2013; 14: 15910-30.

22. Brigger I, Dubernet C, Couvreur P. Nanoparticles in cancer therapy and diagnosis. Adv Drug Deliv Rev. 2012.54: 631-51

23. Tian J, Bai J, Yan XP, Bao S, Li Y, Liang W, et al. Multimodality molecular imaging. IEEE Eng Med Biol Mag. 2008; 27: 48-57.

24. Magonov S, Elings V, Whangbo M-H. Phase imaging and stiffness in tapping-mode atomic force microscopy. Surf Sci. 1997; 375: L385-L91.

25. El-Gogary RI, Rubio Carrero N, Wang JT-W, Al-Jamal WT, Bourgognon M, $\mathrm{Kafa} \mathrm{H}$, et al. Polyethylene glycol conjugated polymeric nanocapsules for targeted delivery of quercetin to folate-expressing cancer cells in vitro and in vivo. ACS nano. 2014; 8:1384-401.

26. Mandal SK, Lequeux N, Rotenberg B, Tramier M, Fattaccioli J, Bibette J, et al. Encapsulation of magnetic and fluorescent nanoparticles in emulsion droplets. Langmuir. 2005; 21: 4175-9.

27. Jarzyna PA, Skajaa T, Gianella A, Cormode DP, Samber DD, Dickson SD, et al. Iron oxide core oil-in-water emulsions as a multifunctional nanoparticle platform for tumor targeting and imaging. Biomaterials. 2009; 30: 6947-54.

28. Maeda $\mathrm{H}$. The enhanced permeability and retention (EPR) effect in tumor vasculature: the key role of tumor-selective macromolecular drug targeting. Adv Enzyme Regu. 2001; 41: 189-207.

29. Chertok B, Moffat BA, David AE, Yu F, Bergemann C, Ross BD, et al. Iron oxide nanoparticles as a drug delivery vehicle for MRI monitored magnetic targeting of brain tumors. Biomaterials. 2008; 29: 487-96.

30. Pulfer SK, Gallo JM. Enhanced brain tumor selectivity of cationic magnetic polysaccharide microspheres. J Drug Target. 1998; 6: 215-27.

31. Ai H, Flask C, Weinberg B, Shuai XT, Pagel MD, Farrell D, et al. Magnetite-Loaded Polymeric Micelles as Ultrasensitive Magnetic-Resonance Probes. Adv Mater. 2005; 17: 1949-52.

32. Mikhaylov G, Mikac U, Magaeva AA, Itin VI, Naiden EP, Psakhye I, et al. Ferri-liposomes as an MRI-visible drug-delivery system for targeting tumours and their microenvironment. Nat Nanotechnol. 2011; 6: 594-602.

33. Ye F, Barrefelt $\AA$, Asem H, Abedi-Valugerdi M, El-Serafi I, Saghafian M, et al. Biodegradable polymeric vesicles containing magnetic nanoparticles, quantum dots and anticancer drugs for drug delivery and imaging. Biomaterials. 2014; 35: 3885-94.

34. Chertok B, David AE, Yang VC. Brain tumor targeting of magnetic nanoparticles for potential drug delivery: effect of administration route and magnetic field topography. J Control Release. 2011; 155: 393-9.

35. Cole AJ, David AE, Wang J, Galbán CJ, Yang VC. Magnetic brain tumor targeting and biodistribution of long-circulating PEG-modified, cross-linked starch-coated iron oxide nanoparticles. Biomaterials. 2011; 32: 6291-301.

36. Chertok B, David AE, Yang VC. Polyethyleneimine-modified iron oxide nanoparticles for brain tumor drug delivery using magnetic targeting and intra-carotid administration. Biomaterials. 2010; 31: 6317-24.

37. Gultepe E, Reynoso FJ, Jhaveri A, Kulkarni P, Nagesha D, Ferris C, et al Monitoring of magnetic targeting to tumor vasculature through MRI and biodistribution. Nanomedicine (Lond). 2010; 5: 1173-82.

38. Fortin-Ripoche J-P, Martina MS, Gazeau F, Ménager C, Wilhelm C, Bacri J-C, et al. Magnetic targeting of magnetoliposomes to solid tumors with MR imaging monitoring in mice: Feasibility. Radiology. 2006; 239: 415-24.

39. Wagstaff AJ, Brown SD, Holden MR, Craig GE, Plumb JA, Brown RE, et al. Cisplatin drug delivery using gold-coated iron oxide nanoparticles for enhanced tumour targeting with external magnetic fields. Inorganica Chimica Acta. 2012; 393: 328-33.

40. Alexiou C, Tietze R, Schreiber E, Jurgons R, Richter H, Trahms L, et al. Cancer therapy with drug loaded magnetic nanoparticles - magnetic drug targeting. J Magn Magn Mater. 2011; 323: 1404-7.

41. Gautier J, Munnier E, Paillard A, Hervé K, Douziech-Eyrolles L, Soucé M, et al. A pharmaceutical study of doxorubicin-loaded PEGylated nanoparticles for magnetic drug targeting. Int J Pharm. 2012; 423: 16-25.

42. Jacques SL. Optical properties of biological tissues: a review. Phys Med Biol. 2013; 58: R37-61

43. Sinusas AJ, Bengel F, Nahrendorf M, Epstein FH, Wu JC, Villanueva FS, et al. Multimodality cardiovascular molecular imaging, part I. Circ Cardiovasc Imaging. 2008; 1: 244-56

44. Lübbe AS, Bergemann C, Riess H, Schriever F, Reichardt P, Possinger K, et al. Clinical experiences with magnetic drug targeting: a phase I study with 4'-epidoxorubicin in 14 patients with advanced solid tumors. Cancer Res. 1996; 56: 4686-93.

45. Shapiro B, Kulkarni S, Nacev A, Muro S, Stepanov PY, Weinberg IN. Open challenges in magnetic drug targeting. Wiley Interdiscip Rev Nanomed Nanobiotechnol. 2015 7:446-57.

46. Cole AJ, Yang VC, David AE. Cancer theranostics: the rise of targeted magnetic nanoparticles. Trends Biotechnol. 2011; 29: 323-32.

47. Mody VV, Cox A, Shah S, Singh A, Bevins W, Parihar H. Magnetic nanoparticle drug delivery systems for targeting tumor. Appl. Nanosci. 2014; 4: 385-92.
48. Kubo T, Sugita T, Shimose S, Nitta Y, Ikuta Y, Murakami T. Targeted delivery of anticancer drugs with intravenously administered magnetic liposomes in osteosarcoma-bearing hamsters. Int J Oncol. 2000; 17: 309-24.

49. Kubo T, Sugita T, Shimose S, Nitta Y, Ikuta Y, Murakami T. Targeted systemic chemotherapy using magnetic liposomes with incorporated adriamycin for osteosarcoma in hamsters. Int J Oncol. 2001; 18: 121-6.

50. Huang Z, Pei N, Wang Y, Xie X, Sun A, Shen L, et al. Deep magnetic capture of magnetically loaded cells for spatially targeted therapeutics. Biomaterials. 2010; 31: 2130-40.

51. Kreuzer M, Kirchgessner M. Endogenous iron excretion. A quantitative means to control iron metabolism? Biol Trace Elem Res. 1991; 29: 77-92.

52. Millet I, Bouic-Pages E, Hoa D, Azria D, Taourel P. Growth of breast cancer recurrences assessed by consecutive MRI. BMC Cancer. 2011; 11: 155. 